\title{
X-ray dark-field phase-contrast imaging: Origins of the concept to practical implementation and applications
}

\author{
Masami Ando ${ }^{\text {a,b,* }}$, Rajiv Gupta ${ }^{c}$, Akari Iwakoshi ${ }^{\mathrm{d}}$, Jong-Ki Kim ${ }^{\mathrm{e}}$, Daisuke Shimao ${ }^{\mathrm{f}}$, \\ Hiroshi Sugiyama $^{\mathrm{a}}$, Naoki Sunaguchi ${ }^{\mathrm{g}}$, Tetsuya Yuasa ${ }^{\mathrm{h}}$, Shu Ichihara ${ }^{\mathrm{d}}$ \\ ${ }^{\text {a }}$ Photon Factory, KEK, Oho 1-1, Tsukuba, Ibaraki 305-0801, Japan \\ ${ }^{\mathrm{b}}$ CROSS, Kamitakatsu 1601, Tsuchiura, Ibaraki 300-0811, Japan \\ ${ }^{\mathrm{c}}$ Department of Radiology, Massachusetts General Hospital and Harvard Medical School, Boston, MA 01778, United States \\ d Clinical Research Center, Department of Pathology, Nagoya Medical Center, Nagoya, Aichi 460-0001, Japan \\ ${ }^{\text {e }}$ Catholic University of Daegu School of Medicine, 3056-6 Daemyung 4 Dong, Nam-Gu, Daegu 705-718, South Korea \\ ${ }^{\mathrm{f}}$ Department of Radiological Technology, Hokkaido University of Science, Sapporo, Hokkaido 006-8585, Japan \\ ${ }^{g}$ Graduate School of Medicine, Nagoya University, 1-1-20 Daiko-Minami, Higashi-Ku, Nagoya, Aichi 461-8673, Japan \\ ${ }^{\mathrm{h}}$ Graduate School of Engineering and Science, Yamagata University, 4-3-16 Jyonan, Yonezawa, Yamagata 992-8510, Japan
}

\section{A R T I C L E I N F O}

\section{Keywords:}

XDFI

Algorithm

Application to pathology

Clinical trial

\begin{abstract}
A B S T R A C T
The basic idea of X-ray dark-field imaging (XDFI), first presented in 2000, was based on the concepts used in an $\mathrm{X}$-ray interferometer. In this article, we review 20 years of developments in our theoretical understanding, scientific instrumentation, and experimental demonstration of XDFI and its applications to medical imaging. We first describe the concepts underlying XDFI that are responsible for imparting phase contrast information in projection X-ray images. We then review the algorithms that can convert these projection phase images into three-dimensional tomographic slices. Various implementations of computed tomography reconstructions algorithms for XDFI data are discussed. The next four sections describe and illustrate potential applications of XDFI in pathology, musculoskeletal imaging, oncologic imaging, and neuroimaging. The sample applications that are presented illustrate potential use scenarios for XDFI in histopathology and other clinical applications. Finally, the last section presents future perspectives and potential technical developments that can make XDFI an even more powerful tool.
\end{abstract}

\section{Introduction}

Wilhelm Röntgen discovered X-rays in late 1895 and published an Xray image of his wife in early 1896 , thereby opening the field of medical imaging. This great achievement was a step forward in medicine. Commemorating the 125th anniversary of this significant advancement, in this article we present a focused review of X-ray dark-field imaging (XDFI), a novel phase-contrast X-ray imaging technique. We review the past 20 years of development of this modality from its inception to its current form.

Motion-based tomography based on simple X-ray projection imaging and fluoroscopy was developed in the early 1930s. Many years later, in the early 1970s, computed tomography (CT) was invented. The advent of flat-panel detectors in the 1990s enabled tomosynthesis, which has since become the dominant modality in diagnostic mammography. These X-ray imaging modalities are based on differences between the Xray absorption by various body tissues. As a result of these inherent differences, the X-ray imaging weights of human organs differ from bones $(14.3 \%)$ to blood vessels $(7.8 \%)$ to soft tissues (7.9\%). Consequently, traditional absorption-based X-ray imaging provides sharp image contrast for some tissues. For example, bones have higher absorption than soft tissue so that they can be readily visualized by absorption contrast. However, imaging soft tissue often requires the use of extrinsic contrast agents such as high-Z elements like I (iodine), Gd (gadolinium), and Ba (barium). These agents selectively induce contrast in organs that contain small blood vessels (such as digestive organs) and relatively thick blood vessels for better visualization with standard X-ray imaging techniques. X-ray imaging of lungs can be used to distinguish

\footnotetext{
* Corresponding author at: Photon Factory, KEK, Oho 1-1, Tsukuba, Ibaraki 305-0801, Japan.

E-mail addresses: ando@post.kek.jp (M. Ando), RGUPTA1@mgh.harvard.edu (R. Gupta), akari@med.nagoya-u.ac.jp (A. Iwakoshi), jkkim@cu.ac.kr (J.-K. Kim),

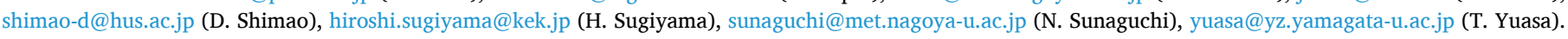




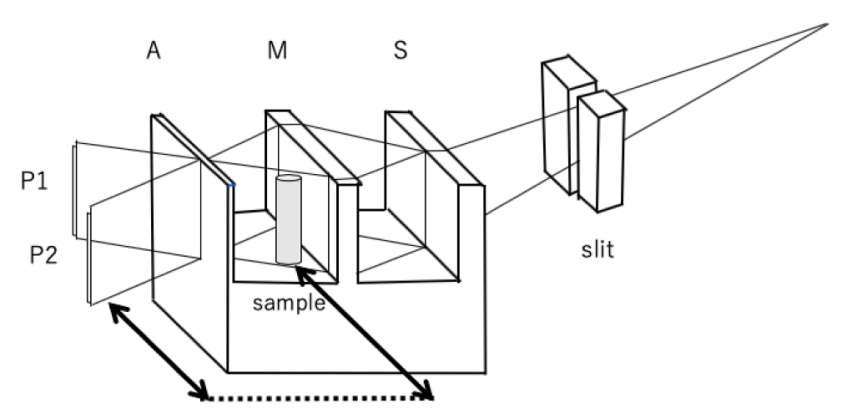

Fig. 1. Sketch of the Bonse-Hart LLL type X-ray interferometer. The structure is monolithically cut out from a high-quality float-zone FZ grown silicon single crystal. The splitter $S$ and mirror $M$ are thick enough to make the outgoing beams nearly parallel using anomalous absorption phenomenon (Borrmann effect). The distance between $S$ and $M$ is almost the same as that between $M$ and analyzer A. Thus, two phase-contrast images P1 and P2 are produced by superimposing two beams from $\mathrm{M}$ one the image through sample and the other reference beam both of which are analyzed in A.

stroma containing fluid (which appear black) from healthy regions containing air (which appear white).

Despite the use of contrast agents, X-ray imaging cannot easily visualize soft tissues. To address this issue, magnetic resonance imaging (MRI) and ultrasound (US) have been developed, providing us with very high contrast. However, unfortunately these modalities offer relatively very low spatial resolution except for a special part of body such as finger, hindering their application to early disease diagnostics.

As an alternative approach to imaging soft tissues, refraction-based $\mathrm{X}$-ray neutron imaging has been developed to realize phase-contrast techniques. Ando and Hosoya world first presented a novel X-ray imaging technique for biomedical specimens [1] at the International Conference on X-Ray Optics and Microanalysis held in Osaka in 1971. Their tool to visualize biomedical specimen was an X-ray interferometer that was devised by Bonse and Hart in 1965 [2]; the presentation was printed in the conference proceedings [1] in 1972. Later, two Russian groups achieved refraction-based imaging: Shilstein et al. at the Kurchatov Institute in Moscow reported crystal-based phase-contrast imaging using neutron beams in 1989 [1-4], and Ingal and Beliaevskaya in St. Petersburg reported the use of Bragg-Laue optics in 1995 [5]. Wilkins of Australia developed X-ray imaging using another method involving crystal optics in 1995 [6]. These two studies used laboratory $\mathrm{X}$-ray sources.

In the early 1980s, synchrotron X-ray irradiation emerged as a popular tool for X-ray imaging because of its high fidelity, high firstorder coherence, easy wavelength tunability, and high brilliance. Since then, a variety of phase-contrast imaging modalities have been competitively developed, and X-ray refraction-based imaging has been extensively explored. Around the 1990s, X-ray imaging trials relying on high first-order coherence were conducted [7]. For example, Chapman presented the idea of diffraction enhanced imaging (DEI) in 1996 [8], and Momose proposed X-ray interferometry-based CT in 1996 [9]. Several medical imaging centers were founded around the world such as the Photon Factory [10], Trieste Synchrotron [11], Grenoble Synchrotron [12], SPring-8 [13], Swiss Light Source [14], Canadian Light Source [15], Australian Synchrotron [16], and Shanghai Synchrotron [17]. Several synchrotron-based X-ray imaging centers were also formed outside synchrotron labs, such as Helsinki [18] and King's College [19].

In this review, we focus on one of the above mentioned novel X-ray refraction-contrast imaging named XDFI that was published in 2002 [20]. This is based on its proactive works [21-24]. Since then, our group has been developing both instrumental components as well as medical applications. XDFI offers a spatial resolution approaching $8.5 \mu \mathrm{m}$ [25], thus enabling X-ray histopathology in projection and tomographic domains. Herein, we describe the fundamental principles of phase-contrast $\mathrm{X}$-ray imaging, the basic setup and advantages of XDFI, and its recent

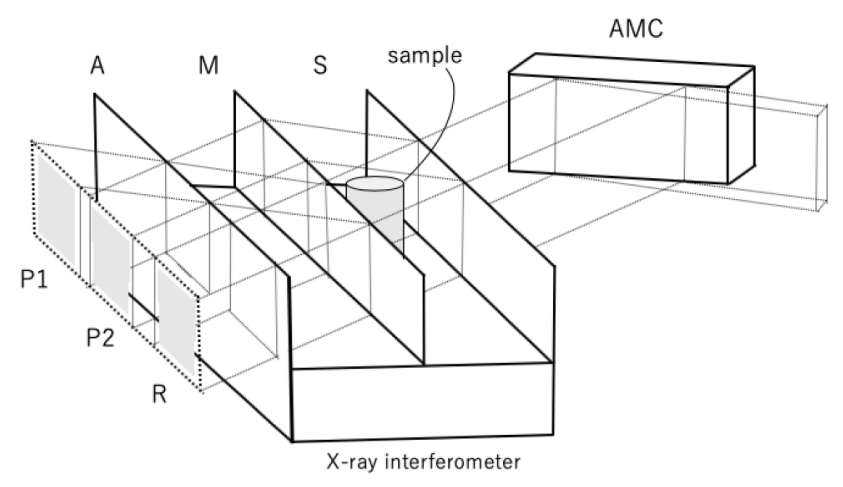

Fig. 2. X-ray interferometer combined with synchrotron radiation. Unlike the classical X-ray interferometry setup, this setup can provide an incident beam to the X-ray interferometer that is both highly collimated and wide enough to be able to image a whole specimen. This design can be used to readily form a refraction-based image $\mathrm{R}$.

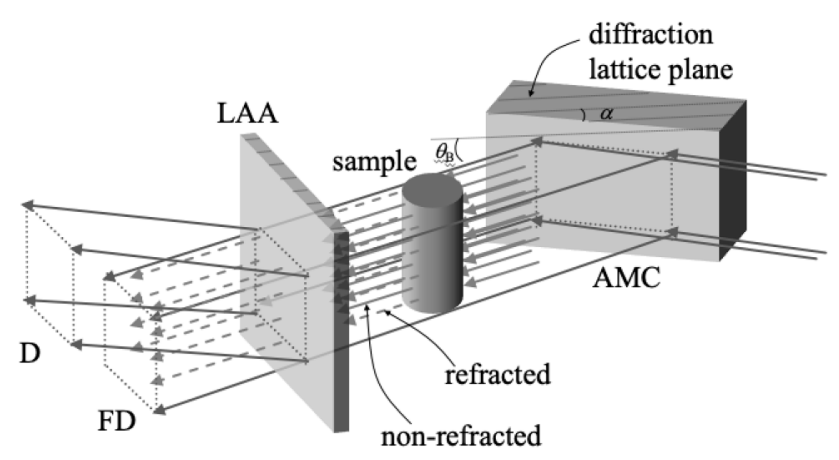

Fig. 3. Scheme of XDFI. The system comprises AMC and LAA. A sample is positioned between the AMC and LAA. The AMC supplies a plane wave incident onto the specimen. Refracted X-rays are angularly analyzed by the LAA. Images are detected by an imaging device.

applications in the histopathologic analyses of various tissue specimens. Finally, we discuss future directions for the further development of XDFI.

\section{Birth of the concept of XDFI}

The idea of XDFI started from the well-known Bonse-Hart LLL (Laue Laue Laue)-type X-ray interferometer (Fig. 1) [2] that was devised before the advent of synchrotron radiation. To conduct X-ray imaging with high spatial resolution, a $50 \mathrm{kV}, 1 \mathrm{~mA} \mathrm{X}$-ray microfocus generator with an apparent focus size of $50 \mu \mathrm{m}$ by $50 \mu \mathrm{m}$ was commonly used. That system guaranteed a spatial resolution around $1 \mu \mathrm{m}$ but required an approximately $12 \mathrm{~h}$ exposure time (i.e., overnight) in scanning mode to obtain 2-dimentional image so that the horizontal beam width was limited to $50 \mu \mathrm{m}$ using a slit. Therefore, to image larger samples, the imaging device, nuclear plate, and sample had to be simultaneously scanned, forming two nearly identical images (P1 and P2) as shown in Fig. 1.

In the mid-1980s, storage ring-based synchrotron radiation sources providing X-ray photons were developed. They offered very high power, so the exposure could be reduced to the order of one second compared to that by a laboratory X-ray source which needs approximately $10^{4}-10^{5}$ times of exposure time. An asymmetrically cut monochromator (AMC) could be used to make the outgoing beam a plane wave so that $S$ and $M$ in the X-ray interferometer does not need thick blades and, at the same time, AMC expands the lateral beam size, thereby eliminating the need to scan the specimen to capture a complete image, as shown in Fig. 2. Using this design, not only the classical images, P1 and P2, but also a 
new image $\mathrm{R}$ emerged.

Building on this design, a simplified X-ray optical system comprising an AMC and a Laue angle analyzer (LAA) [20] was proposed. This concept, termed XDFI, is illustrated in Fig. 3. A sample is placed between an AMC and a LAA with the same angular resolution as the angular divergence of X-rays from the AMC. An X-ray beam sufficiently collimated by the AMC is incident on a sample. In this case, the precise Bragg angle of the incident X-ray beam from the AMC to the LAA is adjusted at the Bragg condition.

Thus, the refracted X-ray component from the sample will deviate from the precise Bragg condition of the LAA. The component that is not refracted by the sample is diffracted by the analyzer as well at different angle that will make a different contrast. In this way, the analyzer separates the refracted and non-refraction components of the image. Hence, a dark-field image is thus obtained in the FD (forward diffraction) direction of the analyzer, and a bright-field image can be obtained in the $\mathrm{D}$ (diffraction) direction as well.

\section{Setups for phase extraction}

An X-ray propagating through a material is refracted according to the material's complex refractive index, $n=1-\delta+i \beta$, where $\delta$ and $\beta$ describe the phase shift and absorption imparted by the material, respectively. The image contrast in the conventional X-ray imaging systems that are widely used in clinical practice is based on absorption by different types of tissues, so the resulting image is primarily based on $\beta$. Although such images provide high contrast for bones and calcifications, which comprise high-Z elements, they provide low contrast for soft tissues that are constituted by low-Z elements such as $\mathrm{H}, \mathrm{C}, \mathrm{N}$, and $\mathrm{O}$. Conversely, image contrast based on $\delta$, commonly known as phasecontrast imaging, addresses this deficiency because $\delta$ is three orders of magnitude larger than $\beta$. The $\delta$ values for soft tissues are typically on the order of $10^{-7}$ with X-ray energies around several tens of $\mathrm{keV}$. Therefore, according to Snell's law, an X-ray beam emanating from a soft tissue sample is refracted by about $10^{-7}$ rad off the incident direction. Such a small angular deviation in the straight-line path of X-ray is too small to detect using traditional imaging setups.

The previously proposed detection techniques can be categorized into crystal interferometry, inline holography, grating interferometry, and angle-analyzer-based methods. In X-ray interferometry, phase contrast is abstracted from interferograms generated by the signal beam emanating from the object and the reference beams using the Bonse-Hart interferometer [2] made of a Si single crystal [26-28]. In inline holography, coherent X-rays are generated from a synchrotron source, which has high first-order coherence, and the interference between deviated beams and straight beams is measured by an X-ray camera located a fixed distance from the sample [7,29,30]. Grating-based imaging involves the use of grating optics between the source and sample and can be performed using a laboratory X-ray source or a synchrotron source $[31,32]$. Angle-analyzer-based methods employ a single crystal plate as an angular analyzer to obtain the refraction angle [5,8,33,34]. All of these techniques have been extended to tomographic imaging. Inline holography and grating interferometry have been used for clinical applications $[35,36]$ because the former has a relatively simple system configuration, and the latter can be implemented with an X-ray tube. Conversely, X-ray interferometry and analyzer-based imaging methods are mainly used for tissue samples because they can provide highdefinition images with resolutions of no more than several tens of micrometers.

\section{Experimental setup of XDFI}

\subsection{Detailed theory underlying XDFI}

Because the refractive index of X-rays is about $10^{-6}$ rad or smaller, an optical device for dividing the X-rays refracted by a sample should have

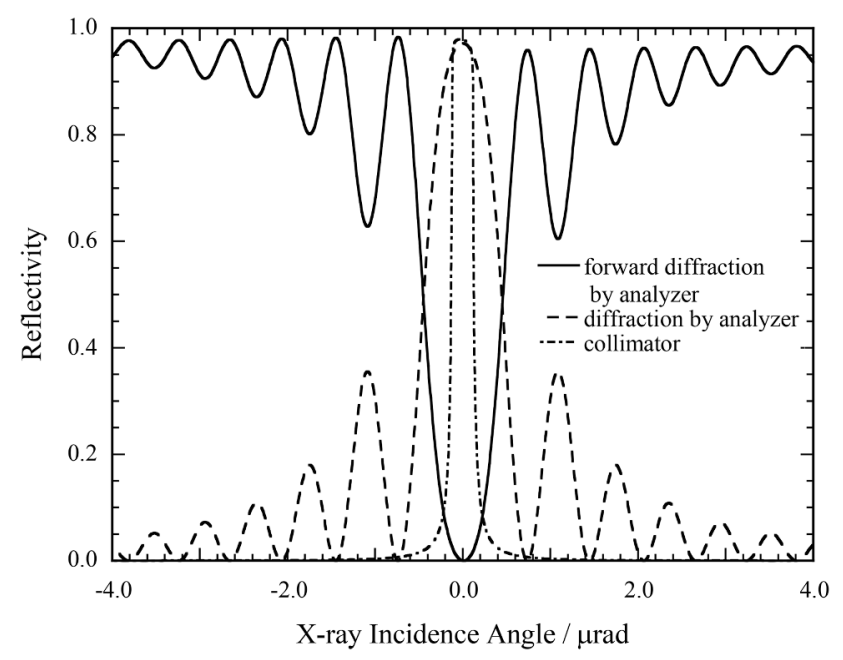

Fig. 4. Rocking curves for the incident beam from the AMC (dash-dotted line), FD (solid line), and D (dashed line) from the LAA, respectively. FD and D were calculated using the formula (2) that ignores the X-ray absorption in a silicon crystal. Under certain conditions, such as $35 \mathrm{keV}$ X-ray energy, 440 diffraction index, and $170 \mu \mathrm{m}$ LAA thickness, the minimum reflection of the FD reaches zero at a zero incidence angle and the maximum reflection of $\mathrm{D}$ reaches almost $100 \%$. The experimental rocking curves of FD and D result from the convolution of beam from AMC with FD and D, respectively, so that the subsidiary peaks attached to the main FD and D profiles will be almost eliminated. The full width at half maximum (FWHM) of the collimator beam from AMC is $2.5 \times 10^{-7} \mathrm{rad}$, so the FWHM of FD and D will increase by only $2 \%$.

an angular resolution of $10^{-6}$ rad or higher. The quality of the crystals used for the AMC and LAA in an XDFI device directly affects the quality of the acquired image. Therefore, the deviation of both the lattice $\mathrm{d}$ spacing, $\Delta \mathrm{d} / \mathrm{d}$, and lattice inclination, $\Delta \Theta$, of the crystal should be $10^{-7}$ or less in the optical device, which is one order of magnitude smaller than angular resolution required for imaging. The X-ray diffraction phenomenon in such a highly perfect FZ crystal can be elucidated by the X-ray dynamical diffraction theory based on multiple reflections [37-40].

The AMC and LAA in an XDFI system use the "Bragg case" and "Laue case" of X-ray dynamical diffraction theory, respectively. In the Bragg case in the AMC the diffracted beam is reflected from the front side of the crystal (i.e., the side through which the X-rays are incident); the effect is similar to a visible light beam being reflected by a mirror. In this case, the diverging X-rays can be further collimated by using a condition called asymmetric reflection, in which the crystal surface and the diffraction lattice plane are not parallel as seen in Fig. 3. The reflection width, $\omega$, in asymmetrical reflection can be described as follows:

$\omega=\omega_{0} \sqrt{\frac{\sin \left(\theta_{\mathrm{B}}-\alpha\right)}{\sin \left(\theta_{\mathrm{B}}+\alpha\right)}}$

where $\omega_{0}$ is the reflection width under the symmetrical condition, $\theta_{\mathrm{B}}$ is the Bragg diffraction angle, and $\alpha$ is the angle between the crystal surface and the diffraction lattice plane. When $\theta_{\mathrm{B}}=10.6^{\circ}$ and $\alpha=10.2^{\circ}, \omega$ can be made approximately one order of magnitude smaller than $\omega_{0}$; because $\omega_{0}$ is usually about $10^{-6} \mathrm{rad}$, the X-ray beam can be collimated to a $\omega$ value of about $10^{-7} \mathrm{rad}$. In the Laue case used for the LAA, both the transmitted and diffracted beams proceed to the back side of the crystal (the side opposite from the surface on which the X-ray beam is incident). The diffraction lattice plane is generally offset at a large angle relative to the crystal surface, and an angle of $90^{\circ}$ would cause symmetric reflection. Thus, the symmetrical reflection Laue case is usually used for LAAs in XDFI.

The Laue case differs significantly from the Bragg case in that the intensity of both the transmitted and diffracted beams oscillate 


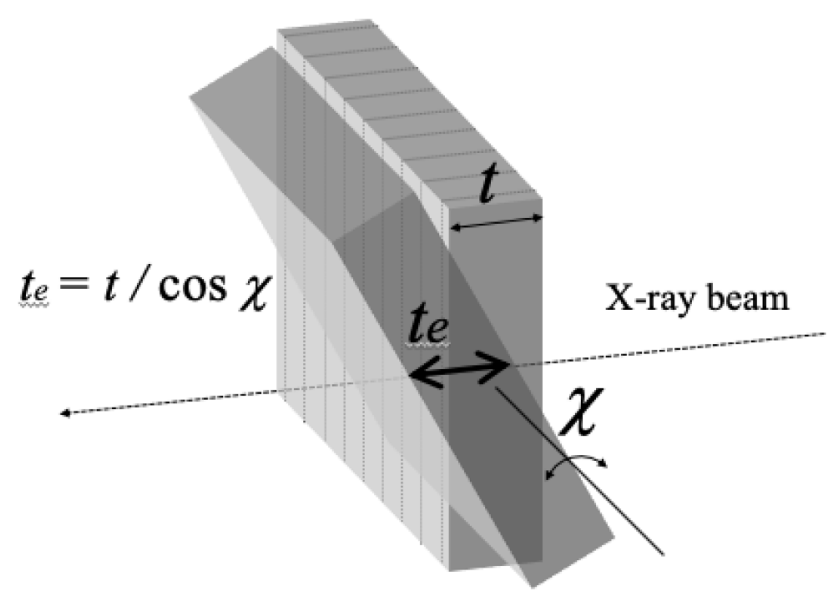

Fig. 5. In order to make the X-ray path length $t$ longer $t_{e}$ the LAA can be rotated around the vertical axis perpendicular to the diffracting planes by $\chi$.

depending on the X-ray incidence angle. This intensity oscillation will follow a rocking curve as follows :

$\mathrm{lo}(\mathrm{W})=\sin ^{2}\left(\mathrm{t} \pi \sqrt{1+\mathrm{W}^{2}} / \wedge\right) /\left(1+\mathrm{W}^{2}\right)$

where $\mathrm{W}$ is the angular parameter, $t$ is the thickness of the LAA, Here, $\Lambda$ $=\lambda \cos \theta_{B} P|| \chi_{G} \mid$, where $\lambda$ is the $\mathrm{X}$-ray wavelength, $\theta_{\mathrm{B}}$ is the Bragg angle, and $\chi_{G}$ is the susceptibility. Further, $\chi_{G}=-r_{e} \lambda^{2} F_{G} / \pi V_{G}$, where $r_{e}$ is the classical radius of an electron, $F_{G}$ is the crystal form factor, and $V_{C}$ is the volume of a unit cell of the crystal. This is called Pendellösung oscillation (herein referred to as the pendel beat) and is known as a typical phenomenon in X-ray dynamical diffraction [37-40].

Fig. 4 shows diffraction profiles (in case of accounting for the absorption) of a Si 440 analyzer at $35 \mathrm{keV}\left(\theta_{\mathrm{B}}=10.6^{\circ}\right)$. The thickness of the analyzer is $172.2 \mu \mathrm{m}$. The output diffraction profile from the collimator AMC with $\alpha=10.2^{\circ}$ is also shown in Fig. 4. These diffraction profiles were calculated using XOP 2.4 software [41].

The pendel beat has an inverse phase between the FD and D beams; this feature is used by the LAA. Furthermore, the period and phase of the pendel beat vary with the thickness of the analyzer crystal. Therefore, it is necessary to adjust the thickness of analyzer crystal so that the transmitted intensity has a minimum value close to 0 and the diffracted intensity has a maximum value of almost $80 \%$ or higher depending on the X-ray absorption properties of the LAA material itself under the precise Bragg condition at the XDFI. This thickness should be a halfinteger multiple of the pendel-beat period. For example, when the pendel-beat period is $114.8 \mu \mathrm{m}$, thickness of the analyzer crystal should be 1.5 times this period, or $172.2 \mu \mathrm{m}$, and the minimum thickness, at which the precise Bragg-condition intensity of the transmitted beam drops to almost 0 , is 0.5 times the pendel-beat period, or $57.4 \mu \mathrm{m}$. Even if the crystal is thinner than 0.5 times the pendel-beat period, the center intensity does not fall significantly, so the performance of the LAA will deteriorate. The LAA thickness is extremely important in XDFI because, if the thickness is not sufficiently close to a half-integer multiple of the pendel-beat period, the intensity of the FD beam at the precise Bragg condition will not be minimized, and the XDFI condition will not be satisfied. However, it becomes difficult to control the LAA thickness with sufficient accuracy as it becomes thin.

There are two ways to precisely satisfy this condition. The first method involves changing the X-ray energy through $\lambda$ in Eq. (2). The second approach is to lengthen the X-ray path inside the LAA by inclining the LAA to adjust the effective thickness of LAA $t$ in Eq. (2) by rotating it about the diffraction vector, as shown in Fig. 5. When the actual crystal thickness is $t$ and the rotation angle is $\chi$, the effective thickness, $t_{e}$, is expressed by the following formula,

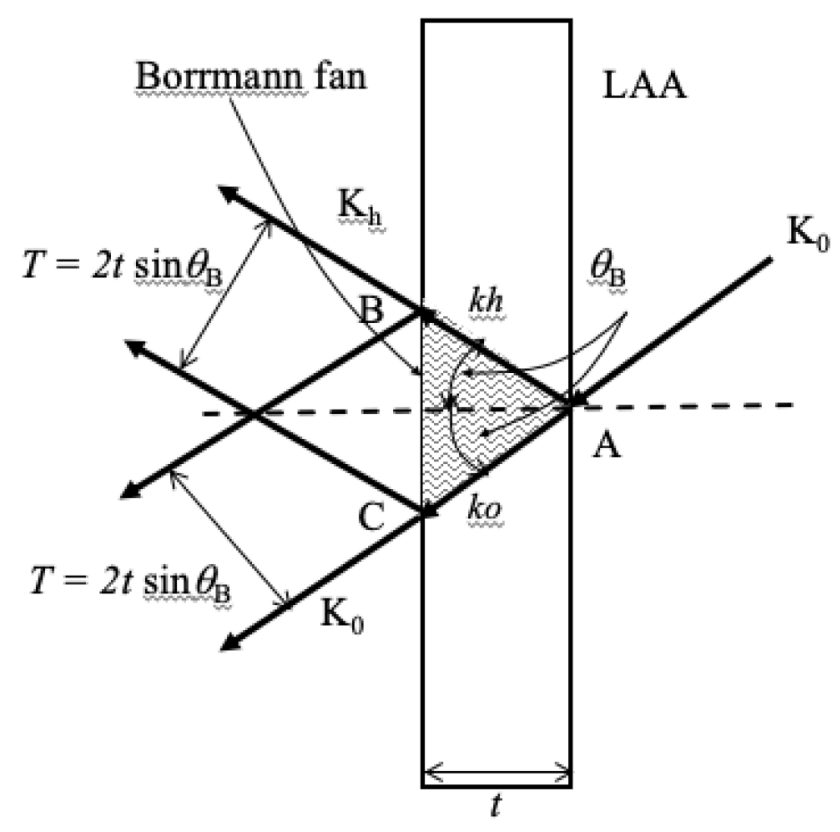

Fig. 6. Schematic drawing showing the relationship between the Borrmann fan, $\triangle \mathrm{ABC}$, and the spatial resolution. If a beam with divergence equal to the rocking-curve width is incident on the apex of the Borrmann fan, the beam inside the LAA propagates fully inside the triangle of the Borrmann fan. Thus, the image blur should correspond to the beam cross-section, $T=2 t \sin \Theta_{B}$ for both the FD and $\mathrm{D}$ beams.

$t_{e}=t / \cos \chi$

Another important factor is the influence of radiation source size on the spatial resolution. First define $\mathrm{x}, \mathrm{y}$ and $\mathrm{z}$ coordinate; we would like to follow the definition in the accelerator science. $\mathrm{z}$ is along the direction from a source point to the optical component same in the electron orbital plane, $\mathrm{x}$ is vertical to $\mathrm{z}$ axis and $\mathrm{y}$ is vertical to $\mathrm{z}$ and $\mathrm{x}$ direction. $\mathrm{X}$-ray imaging has a spatial resolution of $\sigma_{\mathrm{x}, \mathrm{y}}$, which is defined as $\sigma_{\mathrm{x}, \mathrm{y}}=\Sigma_{\mathrm{x}, \mathrm{y}} l / L$, where $\Sigma_{\mathrm{x}, \mathrm{y}}$ is the size of the light source, $l$ indicates the distance between the specimen and the imaging device (such as an X-ray film or CCD camera and $L$ denotes the distance between the light source and the specimen. However, when using synchrotron radiation, $\Sigma_{\mathrm{x}, \mathrm{y}}$ is on the order of several hundred to one thousand $\mu \mathrm{m}$ in 2 standard deviation in Gaussian distribution, $l$ is about several $\mathrm{cm}$, and $L$ is longer than $10 \mathrm{~m}$, so the spatial resolution under this condition is usually about $1 \mu \mathrm{m}$ or less in $\mathrm{y}$ direction while the component along the $\mathrm{x}$ direction at least one order larger.

This relationship determines the XDFI resolution on the plane orthogonal to the incidence plane, which comprises the wave vectors of the incident and diffracted X-rays. More precisely in terms of our typical experimental conditions, $\sigma_{\mathrm{x}}=\Sigma_{\mathrm{x}} l / L=1160 \mu \mathrm{m} \times 69 \mathrm{~cm} / 38 \mathrm{~m}=21 \mu \mathrm{m}$, and $\sigma_{\mathrm{y}}=\Sigma_{\mathrm{y}} l / L=72 \mu \mathrm{m} \times 69 \mathrm{~cm} / 38 \mathrm{~m}=1.3 \mu \mathrm{m}$. These calculations take into account any other conditions such as the effect of placing the point source far away arising asymmetry from the AMC and blurring caused by beam divergence in the Borrmann fan in LAA.

More precisely, regarding the resolution on the scattering plane, blurring corresponding to the thickness $t$ of LAA occurs due to the dynamical diffraction effect of the analyzer. In the Laue case, the wave field spreads over the entire triangle, called the Borrmann fan, which is formed by the incident X-ray and the diffracted wavevector, as shown in Fig. 6. On the dark-field image of FD, the maximum blur amount, T, is represented as $\mathrm{T}=2 t \sin \theta_{\mathrm{B}}$. Therefore, to improve the resolution, it is necessary to make the LAA as thin as possible down to the thickness limit where the X-ray dynamical diffraction theory stands. Please see reference [25] for more details.

Another important factor that should be considered in the system 
design is the installation of this very thin LAA with thickness $<500 \mu \mathrm{m}$ without distortion or bending. One installation method involves the use of a thick, mirror-polished beryllium plate with high X-ray transmittance and sufficient rigidity to hold the analyzer.

Another important consideration is the angular stability of the analyzer. Adequate dark-field images cannot be recorded if the precise Bragg condition of LAA with a $10^{-6}$ rad angular resolution or more cannot be kept for a while for exposure. For this reason, it is necessary to take general anti-vibration measures and to stabilize the temperature of the optical system to about $0.01^{\circ}$ or less to suppress the minute angular changes caused by nonuniform thermal instability. This condition has been achieved by setting up X-ray optical components in an experimental hutch that is installed in the well temperature controlled experimental hall.

\subsection{Fabrication of $A M C$ and $L A A$}

XDFI optics comprises two optical components such as AMC that is a Bragg-case asymmetrically cut crystal block and LAA that is thinner than $2 \mathrm{~mm}$ in Laue case.

Cutting of AMC can be made in laboratory and its mirror polishing has been made outside. Fabrication of LAA has a short history. In the first stage where $40-60 \mathrm{~mm}$ in square with fairly thick crystal thicker than $500 \mu \mathrm{m}$ up to $2.5 \mathrm{~mm}$ thick LAA was prepared cutting, coarse lapping and acid etching was performed at laboratory and mirror polishing was done outside. In the second stage under the condition of thickness below $500 \mu \mathrm{m}$ we have introduced a special design. At present our goal of minimum thickness is $60 \mu \mathrm{m}$ for the X-ray energy of $35 \mathrm{keV}$ and the diffraction index of 440 , and $24 \mu \mathrm{m}$ when the X-ray energy is 20 $\mathrm{keV}$ and the diffraction is 111 that are calculated from the X-ray dynamical diffraction theory using XOP [41]. The reason for that is that these ultimate thicknesses could provide highest spatial resolution around $2-3 \mu \mathrm{m}$.

For four years after we proposed the concept of XDFI in 2002, the LAA was a rectangular shaped plate with approximately $40 \mathrm{~mm}$ by 40 $\mathrm{mm}$ in size holding a uniform thickness of approximately $0.5-2.5 \mathrm{~mm}$. Fig. 6 shows the wave field in the Borrmann fan and demonstrates that the length of the bottom edge $\mathrm{BC}$ of $\triangle \mathrm{ABC}$ can be described as $2 t \tan \Theta_{B}$, which is a function of the LAA thickness $t$ and the Bragg angle $\Theta_{B}$. Because $\mathrm{BC}$ should be correlated with the spatial resolution, we investigated the relationship between $t$ and the apparent spatial resolution.

Results showed clear evidence that decreasing the thickness of the LAA results in higher spatial resolution $[42,43]$. Plate thicknesses of 125.6-2124 $\mu \mathrm{m}$ were investigated, and BC was $794 \mu \mathrm{m}$ when $t$ was 2124 $\mu \mathrm{m}$ and the X-ray energy was $35 \mathrm{keV}$ but $138 \mu \mathrm{m}$ when $t$ was $125.6 \mu \mathrm{m}$ and the X-ray energy was $13.7 \mathrm{keV}$. We asked Hitachi Haramachi Electronics to use ion etching to thin down LAA to $125.6 \mu \mathrm{m}$. The image was much sharper when $t$ was $125.6 \mu \mathrm{m}$ than when $\mathrm{H}$ was $2124 \mu \mathrm{m}$. The spatial resolution could be easily estimated using the Takagi-Taupin equation as approximately $75 \mu \mathrm{m}$ when $t$ was $2124 \mu \mathrm{m}$ and below $6 \mu \mathrm{m}$ when $t$ was $125.6 \mu \mathrm{m}$ so that it is clear that surely improved the spatial resolution [42] but it will need very long time to dig such a deep and wide LAA area; because of this reason unfortunately we stopped this technique.

The ratios between $\mathrm{BC}$ and the measured/calculated spatial resolution were approximately $100 \mu \mathrm{m} / 794 \mu \mathrm{m}=0.094$ and $15 \mu \mathrm{m} / 138 \mu \mathrm{m}=$ 0.13. Thus, the observable spatial resolution can be empirically approximated as $1 / 8$ of $\mathrm{BC}$. This empirical estimation of the spatial resolution should be explained in a future study. This concept represents significant progress toward the challenge of achieving higher spatial resolution (see [25]).

As seen in the previous paragraph, we are interested in fabricating a thinner LAA. Not only to maintain LAA surface atomically flat, to keep thickness equal within $\pm 2 \mu \mathrm{m}$ that is a theoretical limit to keep XDFI condition but also bending of LAA surface due to internal strain that should be released when the crystal has been cut for use has to be as

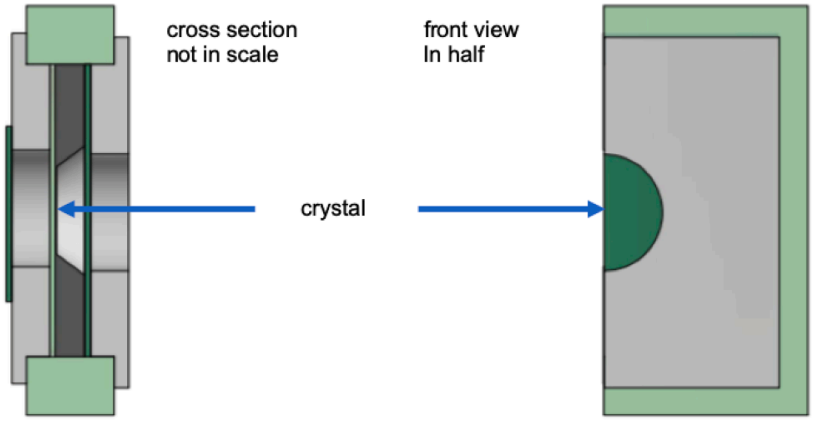

Fig. 7. Cross-section of LAA (not to scale in the left) that has $60^{\circ}$ rise angle at bottom edge of the central part of LAA (shown with arrow). To obtain sufficient stiffness in LAA, it was designed with a thin part in the middle for angular analysis and a thick part with $3 \mathrm{~mm}$ thickness surrounding the thin part to support the crystal. This thickness limit at $3 \mathrm{~mm}$ came from the length of the grinding blade. The thickness of $170 \mu \mathrm{m}$ was chosen so that one can achieve highest spatial resolution under the condition of $35 \mathrm{keV}$ in X-ray energy and 440 diffraction index. The rise angle $\varepsilon=60^{\circ}$ was adopted in this case of $170 \mu \mathrm{m}$. On the right is shown a half of the front view of LAA.

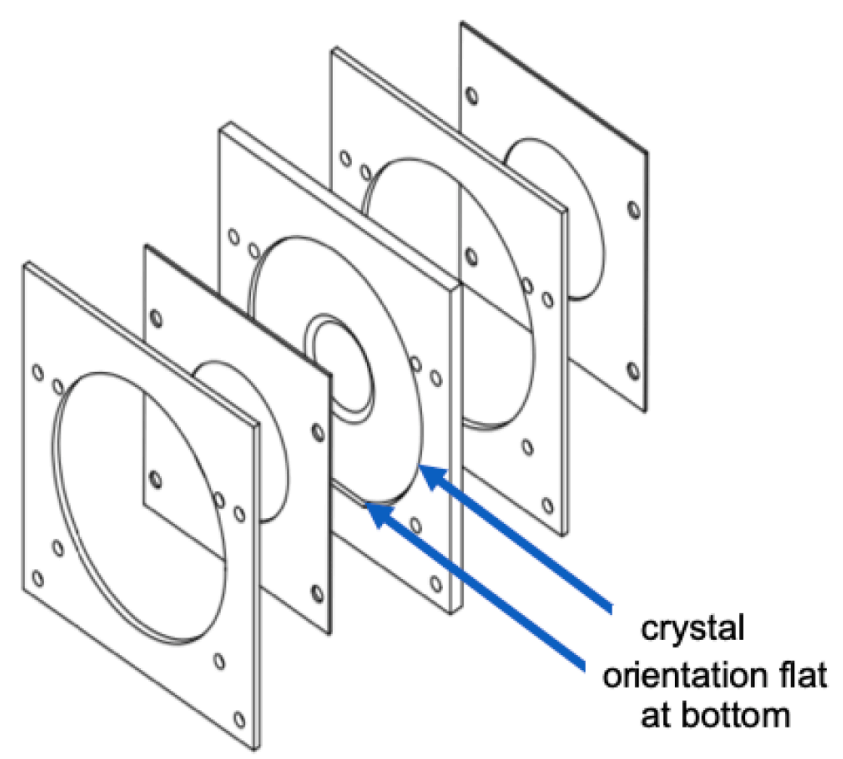

Fig. 8. Sketch of LAA container that mitigates the crystal strain in this setting. The crystal stands vertical such that the diffracting planes of the crystal run vertically because the radiation source has the vertical polarization. In order to have the stability of the LAA it has a flat cut at bottom called orientation flat. The plastic plates shown in Fig. 7 for its cross section are shown in a bird's eye view.

small as possible. Its radius or curvature should be greater than $2-3 \mathrm{~km}$. Thus in order to keep the radius of a thin LAA part as flat as possible we designed its structure as shown in Fig. 7 so that thin part which is surrounded by a $3 \mathrm{~mm}$ thick wall can be flat. Based on the grinder thickness constraint, the maximum wall thickness is $3 \mathrm{~mm}$. In Fig. 7 is shown its cross-section with $170 \mu \mathrm{m}$ thickness. First by mirror-polishing both sides of LAA material a $3 \mathrm{~mm}$-thick blade was prepared. Then, the central part was mechanically ground with the rise angle $\varepsilon=60^{\circ}$ down to $230 \mu \mathrm{m}$ at DISCO in Tokyo. At this stage, the flatness of the thinnest area was \pm 2 $\mu \mathrm{m}$, as measured using a thickness gauge. A hollow upper side of LAA was chemically etched at MIMASU Semiconductor Industry Co., Ltd. in Gunma Prefecture. Surface removal of $60 \mu \mathrm{m}$ seems too deep but we have chosen this number as double of expecting damage layer due to grinding with $30 \mu \mathrm{m}$ for safety. During etching process of LAA to remove the damage layer caused by mechanical grinding at the front side with 
Table 1

Apparatus parameters: see Appendix for Fig. number and reference of publication corresponding to each specimen.

\begin{tabular}{|c|c|c|c|c|c|c|c|c|}
\hline \multirow[t]{2}{*}{ Specimen } & \multirow[t]{2}{*}{$\begin{array}{l}\text { X-ray energy } \\
(\mathrm{keV})\end{array}$} & \multicolumn{3}{|c|}{$\begin{array}{l}\text { X-ray camera : Photonics Science, scintillation } 16 \text { bit CCD } \\
\text { camera }\end{array}$} & \multirow[t]{2}{*}{$\begin{array}{l}\text { Type of } \\
\text { image }\end{array}$} & \multirow[t]{2}{*}{$\begin{array}{l}\text { Number of } \\
\text { projections }\end{array}$} & \multirow[t]{2}{*}{$\begin{array}{l}\text { Absorbed dose } \\
\text { (Gy) }\end{array}$} & \multirow[t]{2}{*}{ Remark } \\
\hline & & pixel size $\mu \mathrm{m}^{\mathrm{H} *} \mu \mathrm{m}^{\mathrm{V}}$ & $\begin{array}{l}\text { Pixel number } \\
\mathrm{H}^{*} \mathrm{~V}\end{array}$ & $\begin{array}{l}\mathrm{FOV} \\
\mathrm{mm}^{\mathrm{H} * \mathrm{~mm}^{\mathrm{V}}}\end{array}$ & & & & \\
\hline TDLU & 35.0 & $8.7 * 6.9$ & $4868 * 3247$ & $8.8 * 6.8$ & $2 \mathrm{D}$ & - & $<0.1$ & XDFI \\
\hline Nipple & 19.8 & $\begin{array}{l}14.8 * 14.8 \text { in } 2 * 2 \\
\text { binning }\end{array}$ & $4868 * 3247$ & $36 * 24$ & $3 \mathrm{D}$ & 600 & 2.4 & XDFI \\
\hline $\begin{array}{l}\text { Benign phyllodes } \\
\text { tumor }\end{array}$ & 35.0 & $7.4 * 7.4$ & $4868 * 3247$ & $36 * 24$ & $2 \mathrm{D}$ & - & $<0.1$ & XDFI \\
\hline DCIS & 35.0 & $6.3 * 6.6$ & $1392 * 1040$ & $8.8 * 6.8$ & $3 \mathrm{D}$ & 900 & $<5.3$ & by DEI optics \\
\hline LCIS & 35.0 & $7.4 * 7.4$ & $4868 * 3247$ & $36 * 24$ & $3 \mathrm{D}$ & 360 & 10.0 & XDFI \\
\hline Knee & 20.0 & $9.0 * 9.0$ & $4008 * 2670$ & $36 * 24$ & TS & 51 & $<0.233$ & XDFI tomosynthesis \\
\hline Finger joint & 20.0 & $\begin{array}{l}14.8 * 14.8 \text { in } 2 * 2 \\
\text { binning }\end{array}$ & $4868 * 3247$ & $36 * 24$ & TS & 51 & $<0.084$ & XDFI tomosynthesis \\
\hline Brain & 17.5 & $\begin{array}{l}14.8 * 14.8 \text { in } 2 * 2 \\
\text { binning }\end{array}$ & $4868 * 3247$ & $36 * 24$ & $2 \mathrm{D}$ & 360 & 10.1 & $\begin{array}{l}\text { A hospital x-rays B } \\
\text { XDFI }\end{array}$ \\
\hline Artery & 31.0 & $\begin{array}{l}14.8 * 14.8 \text { in } 2 * 2 \\
\text { binning }\end{array}$ & $4868 * 3247$ & $36 * 24$ & $3 \mathrm{D}$ & 360 & 1.2 & XDFI \\
\hline Eye ball & 35.0 & $\begin{array}{l}14.8 * 14.8 \text { in } 2 * 2 \\
\text { binning }\end{array}$ & $4868 * 3247$ & $36 * 24$ & $2 \mathrm{D}$ & 360 & 3.0 & XDFI \\
\hline
\end{tabular}

H: horizontal, V: vertical.

$60 \mu \mathrm{m}$ the other bottom side was rinsing with water to suppress etching. Based on our experience, a flatness of $\pm 2 \mu \mathrm{m}$ can be maintained through mechanical grinding and chemical etching. This was verified by measuring the rocking curve, as only fluctuations greater than $\pm 2 \mu \mathrm{m}$ result in significant differences in the minimum value of the rocking curve effect relative to the calculated profile. The minimum value may increase by $20 \%$ because of differences in the LAA thickness.

Readers who are interested in improving spatial resolution and contrast are encouraged to read paper [25]. We attempted to create thinner crystals following the Takagi-Taupin diffraction theory $[39,40]$, which predicts that the highest resolution will be achieved with a thickness of $60 \mu \mathrm{m}$ for a diffraction index of 440 and X-ray energy of 35 $\mathrm{keV}$ and $24 \mu \mathrm{m}$ for a diffraction index of 111 and X-ray energy of $20 \mathrm{keV}$. This thickness could give $2-3 \mu \mathrm{m}$ spatial resolution.

Later, we began investigating an application of XDFI to pathology, which required a FOV of $24 \mathrm{~mm}$ (vertical) by $36 \mathrm{~mm}$ (horizontal). Definitely we adopted a design of thin LAA. Fig. 8 shows a bird's eye view of LAA and its holder made of acrylic plate. A bottom of LAA when it stands has been cut to be able to sit on a horizontal edge of the acrylic holder.

At last we would like to mention about a potential spatial resolution in case of using a Bragg case angle analyzer (BAA) in XDFI optics. Once we tried the BAA to measure the spatial resolution [44]. However this did not provide us with a favorable result. Still we need further interpretation about $\mathrm{X}$-ray wave field to explain the spatial resolution.

\subsection{Apparatus parameters}

Experimental conditions such as X-ray energy, specifications of X-ray camera used for data acquisition, type of images such as $2 \mathrm{D}, 3 \mathrm{D}$ or tomosynthesis and absorbed dose are listed in Table 1.

\section{Extraction of Three-Dimensional (3D) images}

To obtain a phase-contrast CT image (that is, the distribution of $\delta$ ), a set of line integrals of $\delta$ should be measured. As mentioned in the previous section, XDFI systems can measure the angular deviation between the directions of the incident beam and the beam emanating from the sample. In this section, we will the methods used to reconstruct a set of angular-deviation projections. For this purpose, we first introduce the mathematical relationship between the angular deviation and a line integral of $\delta$.

We start with the ray equation, which is a fundamental equation in

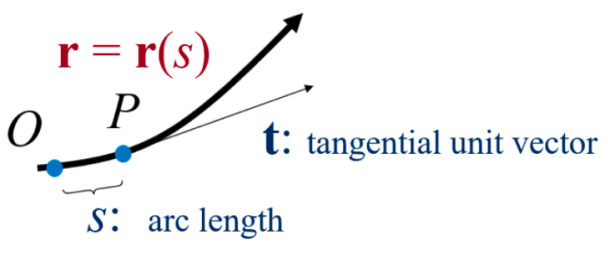

Fig. 9. Ray propagation path represented by the arc length parameter, s, which is the distance from the origin, $\mathrm{O}$, to a point of interest, $\mathrm{P}$, along the path.

optics that represents the relationship between the refractive index and the propagation path: $\frac{d}{d s}(n(\mathbf{r}) \mathbf{t}(\mathbf{r}))=\nabla n(\mathbf{r})$, where $s$ is the arc length along the ray path from the origin to a point of interest, $\mathbf{r}=\mathbf{r}(\mathrm{s})$ is the 3D trajectory representing the ray propagation, and $n(\mathbf{r})$ and $\mathbf{t}(\mathbf{r})$ are the refractive index and the unit tangential vector, respectively, at $\mathbf{r}$ (Fig. 9). Thus, as the analyzer interrupts the photons refracted in the vertical direction, two-dimensional (2D) cross-sections at various horizontal positions would be needed. Therefore, we can regard $\mathbf{t}(\mathbf{r})$ and $\nabla n(\mathbf{r})$ as $2 \mathrm{D}$ vectors in the cross-section of interest. A 3D image is produced by combining the 2D tomographic images reconstructed at different levels.

Substituting $n=1-\delta$ into the ray equation and executing differentiation, we obtain the equation

$(1-\delta) \frac{d \alpha}{d s} \mathbf{v}-\frac{d \delta}{d s} \mathbf{t}=-\nabla \delta(\mathbf{r})$

where $\mathbf{v}$ is the $2 \mathrm{D}$ unit vector normal to $\mathbf{t}$ in the cross-section of interest, $\alpha$ is the angular deviation from the incident propagation direction, and the differential geometry $\frac{d}{d s} \mathbf{t}=\frac{d \alpha}{d s} \mathbf{v}$ is used. Taking the dot product for both sides of Eq. (4) with $\mathbf{v}$ and assuming that $\delta \ll 1$ in the hard X-ray regions, we obtain $\frac{d \alpha}{d s}=-\nabla \delta(\mathbf{r}) \cdot \mathbf{v}$. Introducing $\psi$ as an angle between $\nabla \delta(\mathbf{r})$ and $\mathbf{t}$, the equation can be written as follows:

$\frac{d \alpha}{d s}=-|\nabla \delta| \sin \psi$

Under the paraxial ray approximation that the ray propagates along a line $L$ in the object (because $\delta \ll 1$ ), Eq. (5) is integrated along the line $L$ to obtain

$\int_{L} \frac{d \alpha}{d s} d s=\int_{L} d \alpha=-\int_{L}|\nabla \delta| \sin \psi d s \equiv \Delta \alpha$

where $\Delta \alpha$ is the angular deviation relative to the incident direction, which can be measured using the XDFI imaging setup. 


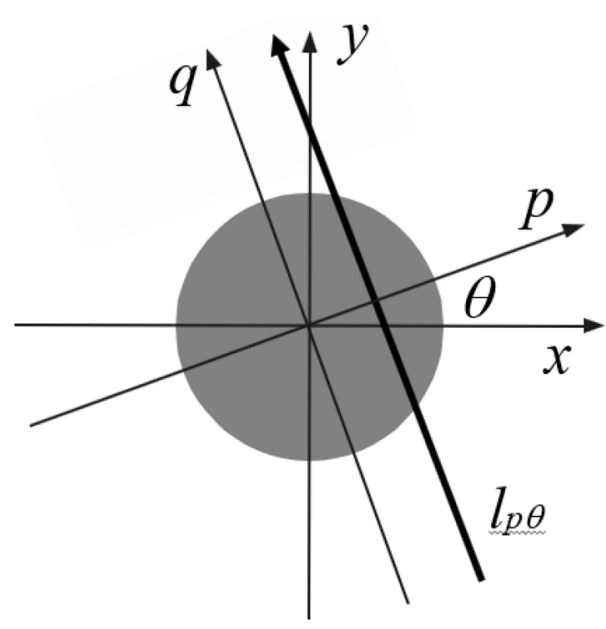

Fig. 10. CT measurement geometry.

Next, we consider the typical coordinate systems for CT measurement. The $x y$ coordinate system is fixed to the object. The $p q$ coordinate system is obtained by rotating the $x y$ coordinate system around the origin by $\theta$ such that the $q$ axis is parallel to the incident ray, as shown in Fig. 10.

According to Eq. (6), we calculate the line integral along line $l_{p \theta}$ (i.e., $\cos \theta x+\sin \theta y=p$ ) to obtain

$\int_{l_{p \theta}} \cos \left(\phi\left(\mathbf{r}_{l_{p \theta}}\right)-\theta\right) d q=\Delta \alpha(p, \theta)$

where $\mathbf{r}_{l_{p \theta}}=(p \cos \theta-q \sin \theta, p \sin \theta+q \cos \theta)$ and $\phi\left(\mathbf{r}_{p \theta}\right)$ is the angle between $\nabla \delta\left(\mathbf{r}_{p \theta}\right)$ and the $x$ axis. Therefore, the angle between $\nabla \delta\left(\mathbf{r}_{l_{p \theta}}\right)$ and the propagation direction, $\psi$, in Eq. (6) corresponds to $\phi\left(\mathbf{r}_{p \theta}\right)-\left(\theta+\frac{\pi}{2}\right)$.

Noting that $\frac{\partial \delta}{\partial x}=|\nabla \delta| \cos \phi$ and $\frac{\partial \delta}{\partial y}=|\nabla \delta| \sin \phi$ and applying $\cos \theta \frac{\partial}{\partial x}+$ $\sin \theta \frac{\partial}{\partial y}=\frac{\partial}{\partial p}$, Eq. (7) can be modified as follows:

$\int_{-\infty}^{\infty}\left(\cos \theta \frac{\partial}{\partial x}+\sin \theta \frac{\partial}{\partial y}\right) \delta\left(\mathbf{r}_{l \theta \theta}\right) d q=\int_{-\infty}^{\infty} \frac{\partial}{\partial p} \delta\left(\mathbf{r}_{p \theta}\right) d q \equiv \Delta \alpha(p, \theta)$

Additionally,

$\int_{-\infty}^{\infty}\left[\int_{-\infty}^{\infty} \frac{\partial}{\partial p} \delta\left(\mathbf{r}_{p \theta}\right) d q\right] \exp (-2 \pi i \rho p) d p$
$=2 \pi i \rho \int_{-\infty}^{\infty}\left[\int_{-\infty}^{\infty} \delta\left(\mathbf{r}_{p \theta}\right) d q\right] \exp (-2 \pi i \rho p) d p$

Therefore, the Radon transform of $\delta$ is the convolution between $\Delta \alpha$ and $\mathscr{F}^{-1}\left[\frac{1}{2 \pi i \rho}\right]$, where $\mathscr{F}^{-1}[\cdot]$ denotes the inverse Fourier transform. It is known that $\mathscr{F}^{-1}\left[\frac{1}{2 \pi i \rho}\right]=\operatorname{sgn}(p)$, where $\operatorname{sgn}(p)=1(p>0), 0(p=0)$, $-1(p<0)$. To reconstruct $\delta$, the convolution reconstruction algorithm that is widely used in conventional CT can be directly applied to $\Delta \alpha(p, \theta) * \operatorname{sgn}(p)$, where * represents convolution. Thus, we can directly obtain a refraction-contrast CT image just by first convolving the angular-deviation projection, $\Delta \alpha(p, \theta)$, with the function $\operatorname{sgn}(p)$ for each $\theta$, then applying the filtered back-projection method to the result.

Here, we introduced the orthodox reconstruction scheme under ideal conditions [45-47]. In practice, the data collection is subject to undesirable conditions, such as a limited number of projections and missing data, so reconstruction must be performed from insufficient projections. Therefore, several reconstruction techniques that are robust against such situations have been proposed [48-52].

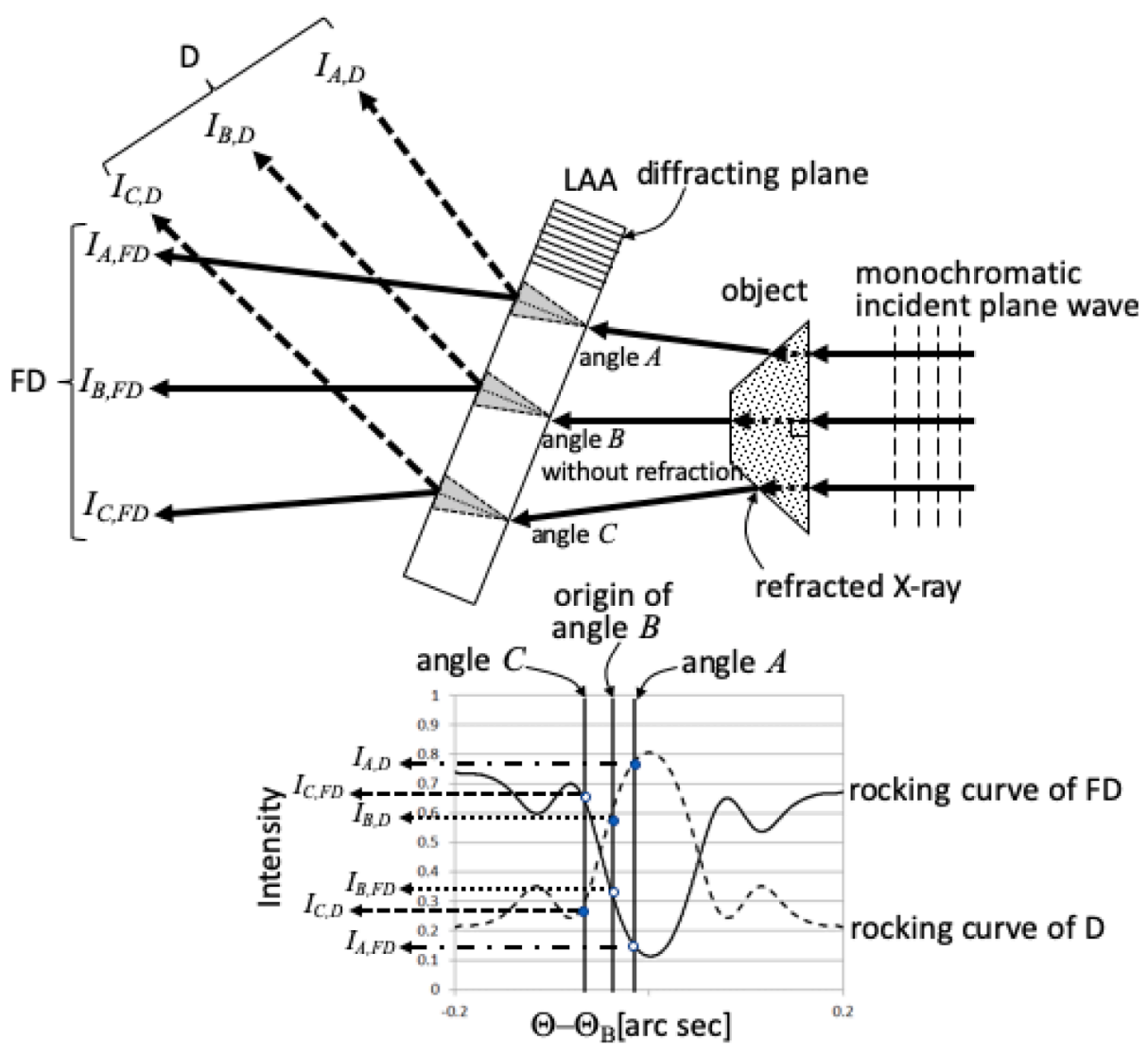

Fig. 11. X-ray monochromatic and parallel beams incident onto a soft tissue specimen. The beam from the tissue comprises a straight beam with a small absorption and a refracted beam produced at the soft tissue boundaries. These are the angles that are analyzed by the LAA so that both FD and D beam may contain absorption and refraction components. In the LAA, the beam is split into a FD beam (straight line) and $\mathrm{D}$ beam (dotted line) following the diffraction theory before they exit LAA. The red color narrow rocking curve at center indicates the beam profile from AMC. If the incident angle is set at the blue dot on the left FD and D rocking curves the biggest dynamic range of black and white intensity of tissue along the vertical axis is available, while the refractive angle position corresponds to the $\mathrm{x}$-axis. 


\section{Crystal-Analyzer-Based phase extraction}

Since the analyzer-based imaging is a method of converting the beam deviation generated by the propagation of an X-ray beam through a sample into intensity information using the slope of a Si single crystalspecific rocking curve, pixel values on the projection image measured by detector represent X-ray intensities. In order to reconstruct the CT representing the distribution of $\delta$ on the sample as mentioned above, these pixel values must be reconverted into refractive angles from X-ray intensities. If the absorption of the sample can be ignored, the refractive angle can be easily obtained from a function of the rocking curve. Otherwise, multiple intensity information must be used to extract the refraction angle to remove an absorption information. The extraction method of refraction angle is different by the directions of the diffraction planes of the analyzer crystal: Bragg-case angle analyzer (BAA) and LAA.

In BAA, the refractive angle can be obtained from the two images obtained at the left and right slopes of the rocking curve of BAA by the following linear calculation [8]:

$\Delta \alpha_{D E I}=R_{\text {Bragg }}^{-1}\left(\frac{I_{\text {left }}-I_{\text {right }}}{I_{\text {left }}+I_{\text {right }}}\right)$

where $R^{-1}$ is the inverse function of the rocking curve of the BAA, and $I_{\text {left }}$ and $I_{\text {right }}$ represent X-ray intensities obtained at the left and right slopes of the rocking curve, respectively. The origin of each slope is usually set at an angle at which the reflectivity is halved.

In LAA, the refractive angle can be obtained from the two images obtained in the directions of the FD and D at the same time as shown in Fig. 11, where the thickness of LAA satisfies XDFI condition as section 4. The extraction method of refractive angle, $\Delta \alpha_{\mathrm{DFI}}$, is as follows: First, each rocking curve of FD and $\mathrm{D}$ is approximated using $N$ th-order polynomial functions:

$R^{T}(\theta)=\sum_{k=0}^{N} a_{k}^{T} \theta^{k}(T=F D, D)$,

where $\theta$ is the angle of deviation relative to the Bragg angle. The coefficients of the polynomial functions, $a_{k}^{T}$, are determined by the leastsquares method from pre-measured intensity data of the rocking curves. The polynomial degree, $N$, is selected as the optimal value according to the X-ray energy or the thickness or the diffraction plane of the LAA. The diffraction intensities can be expressed with respect to $\Delta \alpha_{D F I}$ as follows:

$R^{T}\left(\theta+\Delta \alpha_{D F I}\right)=\sum_{k=0}^{N} a_{k}^{T}\left(\theta_{0}+\Delta \alpha_{D F I}\right)^{k}(T=F D, D)$

where $\theta_{0}$ is the angle of incidence of the X-ray to the LAA when not passing through a sample. The $\mathrm{X}$-ray intensity is attenuated after passing through the sample according to Beer's law, $\exp \left(\int_{L} \mu d l\right)$, where $\mu$ is the line attenuation coefficient, and $L$ is the propagation distance of the $\mathrm{X}$ ray path within the sample. Therefore, the X-ray intensities detected by the cameras behind the forward diffraction and diffraction means are attenuated as follows:

$I^{T}\left(\theta_{0}+\Delta \alpha_{D F I}\right)=R^{T}\left(\theta_{0}+\Delta \alpha_{D F I}\right) \exp \left(-\int_{L} \mu d l\right)(T=F, D)$

From Eqs. (4) and (5), the following $N$-order equation can be obtained [33]:

$\sum_{k=0}^{N}\left(a_{k}^{F}-\frac{I^{F}}{I^{D}} a D k\right)\left(\theta_{0}+\Delta \alpha_{D F I}\right)^{k}=0$

There are $N$ solutions to this equation, of which only one real number can be the solution. If $N$ is less than or equal to 3, the solution can be obtained analytically; at higher orders, numerical methods, such as Newton's method, are used to obtain the solution. To obtain a stable solution by Newton's method, it is important to set an initial value that is

\section{D Visualization methods}

Conventional histological section

- Manual

- Computer-aided

- 3D internal structure microscope

Whole-mount tissue clearing
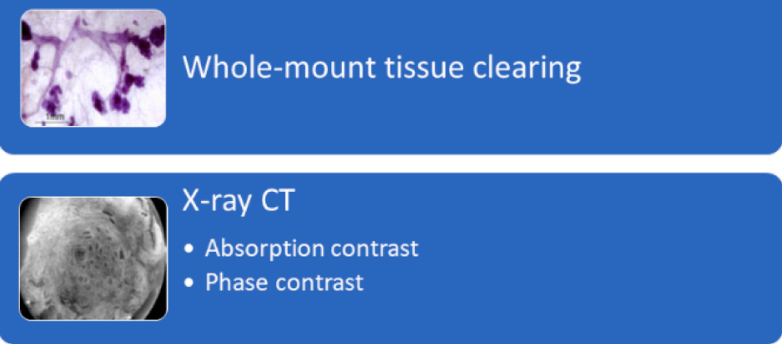

Fig. 12. Different strategies for $3 D$ visualization of specimens at the scale of light microscopy.

close to the solution. In practice, the angle obtained by the first-order expression of Eq. (6) is used as the initial value:

$\Delta \alpha_{\text {init }}=\frac{I_{d e t}^{D} a_{0}^{F}-I_{d e t}^{F} a_{0}^{D}}{I_{d e t}^{F} a_{1}^{D}-I_{d e t}^{D} a_{1}^{F}}$

By performing this process on every pixel of the camera, a projection image representing the refractive angle can be obtained. Then, by substituting $\Delta \alpha_{\mathrm{DFI}}$ obtained from Eq. (8) into Eq. (7), the linear attenuation coefficient can be also obtained. Thus, the refraction and absorption information can be separated from the measurement data obtained from the forward diffraction and diffraction beams at the same time.

\section{Pathological Evaluation: XDFI for non-destructive pathological diagnosis}

\subsection{Overview of cancer diagnostics by conventional pathology}

The most critical portion of cancer treatment is deciding whether a patient has cancer or not. This procedure, called histopathological diagnosis, provides essential information for multiple aspects of patient care including selecting treatment, predicting treatment response, and evaluating prognosis. Usually, a trained pathologist observes the morphology of tissue or cells taken from patients during biopsy or surgery under a light microscope. Diagnostic pathology, established in the late 19th century, has been refined and systematized in the present day, currently involving the traditional method of specimen preparation combined with more sophisticated techniques such as immunohistochemistry or molecular biology. Tissue preparation involves cutting thin slices measuring around $2-3 \mu \mathrm{m}$ in thickness from a tissue block using a mechanical cutter called a microtome. These thin slices are then stained with hematoxylin and eosin (HE). The final cancer diagnosis is determined by a pathologist based on morphological pattern recognition at low magnification and the nuclear and other cytological features at high magnification.

Needless to say, the human body is composed of numerous cells that are 3D. Conventional HE-stained histological sections, however, are usually smaller than $3 \mathrm{~cm}$ by $2 \mathrm{~cm}$ and are 2D. Pathologists examining 2D glass slides intuitively and unconsciously perceive abnormalities in the $3 \mathrm{D}$ structure by mentally reconstructing the $3 \mathrm{D}$ image by integrating random 2D histological slices. However, such mentally generated provisional 3D models are often coarse, incomplete, or even misleading, so the potential to understand the 3D structure of a lesion is limited.

Therefore, a high-resolution 3D model based on sequential slices can 
add value to cancer diagnostics by providing accurate spatial and structural information beyond that of the $2 \mathrm{D}$ images obtained by conventional light microscopy. Such information can reveal structural abnormalities in the tissue and elucidate the tumor configuration.

In this section, we will review various 3D microscopic visualization methods other than X-ray dark-field-computed tomography (XDFI-CT), highlighting the basic principles as well as the cons and pros of each approach (Fig. 12). Comparing XDFI-CT with the other approaches may further shed light on the advantages of XDFI-CT as a non-destructive tool for 3D histology and pathological diagnosis in clinical settings. Furthermore, the combination of 3D geometrical information from XDFI-CT with molecular profiles obtained by other 3D analytical approaches may give rise to powerful new diagnostic techniques.

\section{2. $3 D$ approaches in pathological diagnosis}

\subsubsection{D models of multiple histological sections}

Studies combining tissue dissection and microscopic observation are crucial in clinical diagnostics and translational biomedical research $[53,54]$. Although conventional methods provide an abundance of information about the morphologic changes of cells and tissues, their ability to reveal 3D structures is limited because pathological images are always 2D. In general, pathologists examine an average of 1-2 slides per tissue block. However, as each section is only a small fraction of the 3D resected specimen, under-sampling of the specimen is likely to occur, which can severely reduce the available information compared with what could be obtained by 3D observation. A 3D model of the sectioned tissue has significant potential to enhance the study of normal and disease processes, particularly those involving structural changes such as cancer.

To achieve more comprehensive and efficient visualization of the 3D spatial arrangements of tissue structures, various methods of 3D reconstruction using serial microscopic sections have been presented since the late 1960s and 1970s [55-58]. In the traditional manual reconstruction for reviewing fixed tissue samples, serial slices were projected on tracing paper and the drawings were stacked one upon another in series to examine the 3D architecture of the tissue [56]. However, it is impractical and time-consuming to visualize and analyze numerous slides per sample by conventional microscopy. Moreover, this method is susceptible to intra- and inter-observer variability.

A modern option is to digitize tissue images using a scanner and review the images in dedicated computer software; this method is known as whole-slide imaging (WSI). WSI systems can generate digital images of entire histopathologic glass slides with magnifications close to that of conventional microscopy. High-resolution and wide-field microscopic images can be rapidly captured using WSI to obtain highly detailed information about tissue morphology. Further, WSI can be used to create digital 3D reconstructions from stacks of 2D WSIs. Several methods have been proposed to align multiple 2D WSIs [59-65]. WSI combined with computational technologies also enables quantification of multiple histological parameters, which can provide insights into disease pathogenesis and tissue biology [66]. Moreover, WSI has proved to be particularly suitable for applying deep learning algorithms that can assist humans in analyzing digital images of tissues $[66,67]$. Today, it is possible to generate reconstructed 3D images of a whole tissue section by digitally processing images of serially sectioned tissue, which enables 3D histopathological observation and mapping of lesions. However, limitations persist. One of the major problems encountered in 3D image reconstruction of histology slides is tissue deformation due to the preparation steps, such as sectioning, slice thickness, staining, and mounting, because the quality of the digital images depends on the quality of the original slide [59-63,66,68]. Additionally, it is inevitably difficult to correctly align them with adjacent sections to reconstruct a 3D image. Other slide-related issues include tissue discontinuity, accumulation of scale changes, and the thickness of the glass slides [69].

Moreover, to reconstruct a 3D image by this method, hundreds of glass slides from serial- or step-sectioned tissues are needed, which is a physical and labor-intensive process in most settings. Although further development efforts are expected to enable full-resolution whole-slide visualization in 3D, full-color datasets require much digital memory, so the computational requirements to process and store extremely large image files can be a limitation.

\subsection{2. $3 D$ microscopic internal observation of serially sliced samples}

An alternative destructive observation technique to $3 \mathrm{D}$ tissue reconstruction of individual serial sections is $3 \mathrm{D}$ internal structure microscopy (3D-ISM). 3D-ISM was originally devised by a Japanese researcher, T. Higuchi, in 1990. The technique was developed to observe the internal structures of materials, such as biological samples and industrial materials, on a microscopic scale [70,71]. A 3D-ISM system comprises a computed drive cryotome, a high-sensitivity CCD camera, a rewritable laserdisc recorder, and a computer with a graphic workstation [70]. The biological specimen is fixed in formalin, dipped into an embedding solution, frozen, and positioned under a microscope; then, a very thin layer of the specimen is removed by a computer-controlled slicer. After slicing, the newly created surface is observed by $2 \mathrm{D}$ optical imaging and, if needed, is subjected to various other analyses. Images of each cut surface are recorded by a CCD camera and stored in a digital format. After all of the images are captured, a 3D images of the sample is reconstructed by a full-color ray-casting volume-rendering method [70-72]. This system enables automated investigation of the 3D internal structure of a sample and is compatible with various imaging techniques including fluorescence imaging and whole-mount staining with clearing.

The resolution of a 3D reconstruction obtained by serial sectioning depends on the magnifying power of the microscope used to image each section (in the $x$ - and $y$-planes) and the cutting depth (in the $z$-plane) [71]. As each newly cut bare surface of the specimen is microscopically rough (there are numerous tiny lumps and holes), the diffusion of reflected light by these lumps and holes markedly lowers the quality of the episcopic image [73]. Further, though 3D-ISM yields a full-color, coarse 3D image of the internal structure of a sample, it is limited because it is not possible to observe a region for a second time after the sample has been cut. Moreover, this destructive observation technique is not suitable for samples that are difficult to obtain or should be retained after imaging.

\subsection{3. $3 D$ visualization of intact samples by whole-mount tissue clearing}

For routine histopathology, surgically resected 3D tissue samples are taken from a patient then chemically fixed to preserve the tissue structure. Section-based imaging methods are limited by inevitable discontinuity caused by tissue sectioning; they cannot be used for perfectly continuous observation of the tissue structures. Hence, non-destructive techniques for high-resolution 3D imaging and analysis on the microscopic scale are under investigation.

Although optical imaging is one of the most practical ways to collect information from a tissue sample, tissues consist of various substances with different optical properties, and some scatter or absorb light [74]. The resulting tissue opacity is one of the major obstacles hindering our ability to achieve better optical imaging. To this end, 3D imaging techniques that involve tissue-clearing to minimize light scattering and absorption are being developed. These techniques increase the transparency of a tissue sample so that light can illuminate cells deep within the sample. Efficient tissue clearing contributes to better imaging depth and contrast and is an important step toward achieving whole-body and whole-organ 3D imaging. A tissue-clearing method based on an organic chemical cocktail (a mixture of benzyl alcohol and methyl salicylate) was first reported more than a century ago [75], and remarkable advances have been made in the field since then [76-78]. Current tissueclearing techniques can be classified into three major groups: hydrophobic reagent-based methods, hydrophilic reagent-based methods, and hydrogel-based methods $[76,77,79]$. Recently, several scalable tissueclearing protocols with excellent clearing performance that can be 
used on large tissue blocks or whole organs have been developed $[78,79]$.

Tissue clearing enables the acquisition of volumetric images that can provide detailed information about the internal landscape of a tumor. $3 \mathrm{D}$ volumetric images can be obtained by various optical microscopy techniques such as confocal fluorescence microscopy, multiphoton fluorescence microscopy, and light-sheet fluorescence microscopy (LSFM), which can be used to visualize large tissue samples [80,81]. Additional whole-organ structural information can be obtained by nuclear staining or immunohistochemistry and combined with optical images by registration and alignment of multiple whole-organ images $[78,79]$. These results demonstrate the potential for systematic quantification and analysis of biological information by whole-organ 3D staining and the subsequent computational reconstruction [82]. These advancements are expected to enable the practical implementation of this technology and, ultimately, improve cancer diagnosis and better inform cancer therapy.

While these methods may have advantages for 3D imaging compared with destructive methods, they are potentially limited as they introduce varying combinations of increased complexity, reduced speed, lower resolution, and shorter penetration depth. Furthermore, there is a concern that the chemical clearing agents may cause cell damage such as RNA degradation [78].

\subsubsection{Application of XDFI to conventional histopathology}

CT techniques are the most frequently applied 3D imaging methods in clinical practice. X-ray CT can be used to directly visualize 3D structures as consecutive slice images without destroying the tissue. This technique has demonstrated powerful capabilities in medical diagnosis in clinical practice, especially for analyzing tissues, such as bone, that exhibit sufficient contrast even with low density resolution.

Clinical soft tissue imaging using traditional X-ray CT is based on the absorption or attenuation differences between tissues as the primary contrast mechanism [83]. However, biological soft tissues with high water contents exhibit similar X-ray absorption rates; therefore, there is inadequate contrast between different types of soft tissue under this imaging modality [84]. Consequently, conventional CT analysis is unsuitable for identifying the attenuation associated with small tumors from surrounding healthy tissue unless they are calcified, are causing structural distortion, or have dense cell packing [85]. Thus, iodinated contrast agents must be administered to improve the image quality [86], or alternative imaging techniques, such as MRI, must be used $[87,88]$.

Recently, technological advances have led to new developments in biological soft tissue imaging using X-rays, including phase-contrast CT, sample staining with heavy metals, nanoparticle labeling of cells, X-ray "water window" imaging, and dual-energy CT [89]. The potentiality of laboratory and synchrotron CT imaging is increasing [90]. XDFI-CT $[25,52]$ is one type of phase-contrast CT scanning based on observing refracted X-rays. This innovative imaging modality can be used to obtain 3D images of soft tissue non-destructively with contrast similar to that of low-magnification histopathological imaging without a contrast agent [34]. Previous studies using a variety of specimens (such as breast tissue with cancer, joints with articular cartilage, and ex-vivo human eye specimens) showed that XDFI-CT is more effective in characterizing anatomical features, articular pathology, and neoplastic disease than conventional absorption-based images [34]. XDFI-CT was also found to be useful for exploring the microanatomical structure of human tissue, and it was almost equivalent to low-magnification light microscopy of conventional HE-stained histological sections [91-93]. In recent years, the accuracy of XDFI-CT has been improved, reaching a spatial resolution of $10 \mu \mathrm{m}$ within a measurement time of $1 \mathrm{~h}$ [94]. These preliminary studies suggest that XDFI-CT may ultimately be used to explore the microanatomy of various soft tissues and organs.

Non-destructive pathological diagnosis methods can be used to acquire $3 \mathrm{D}$ data that are typically difficult to obtain using traditional pathology techniques. Therefore, these techniques are expected to enable the comprehensive detection of 3D morphological abnormalities in a high-throughput manner. To use a histopathological sample on a glass slide in an image classification system, it must first be converted to a digital image using an instrument such as a virtual slide scanner. Conversely, images from X-ray CT can be readily used for various digital image analysis methods. Thus, this new technology is expected to be highly beneficial for not only conventional qualitative histopathological diagnosis but also quantitative image classification. Accurate 3D representations of tumor architecture could also benefit cancer diagnosis. Further, if structural differences between healthy tissue and tumor tissue can be visualized, comparing this information with the histopathological analysis would provide a better understanding of the 2D histopathological image; this is another promising application.

Two problems need to be resolved before the clinical application of XDFI-CT: XDFI requires special X-ray beams generated in a large synchrotron radiation facility and requires a large radiation dose. At present, XDFI-CT is an experimental method and has been tested on a limited number of subjects. The application of this method to routine histopathological evaluation for primary screening would help guide decision making by highlighting suspicious areas in unstained tissue for further sampling and analysis. The obtained information could especially help study and quantify 3D patterns of tumor growth. The 3D images could also be used to determine the invasion depths of tumors such as follicular thyroid neoplasms and prostatic neoplasms. Such additional information could help reduce unnecessary procedures.

\section{Oncologic evaluation}

Analyzer-based phase-contrast X-ray imaging can be used to obtain images of soft tissues with quality comparable to that of low-power light
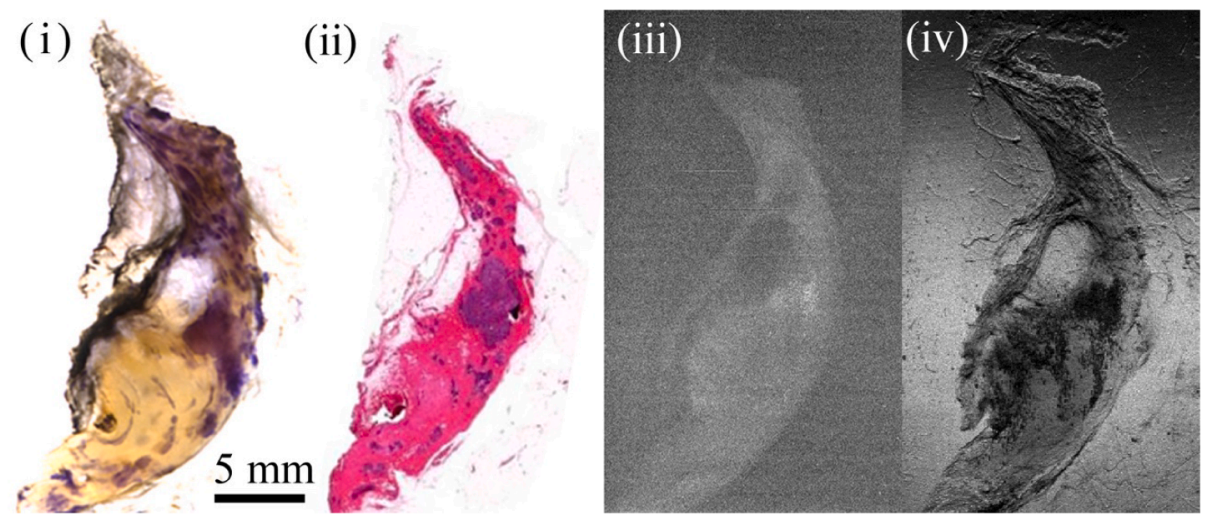

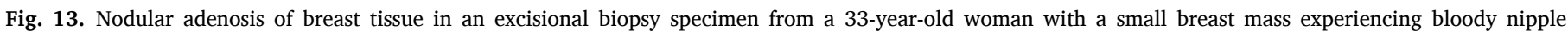

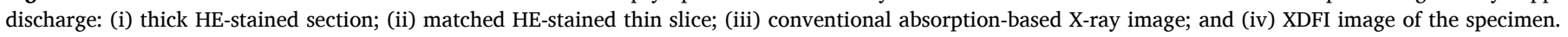




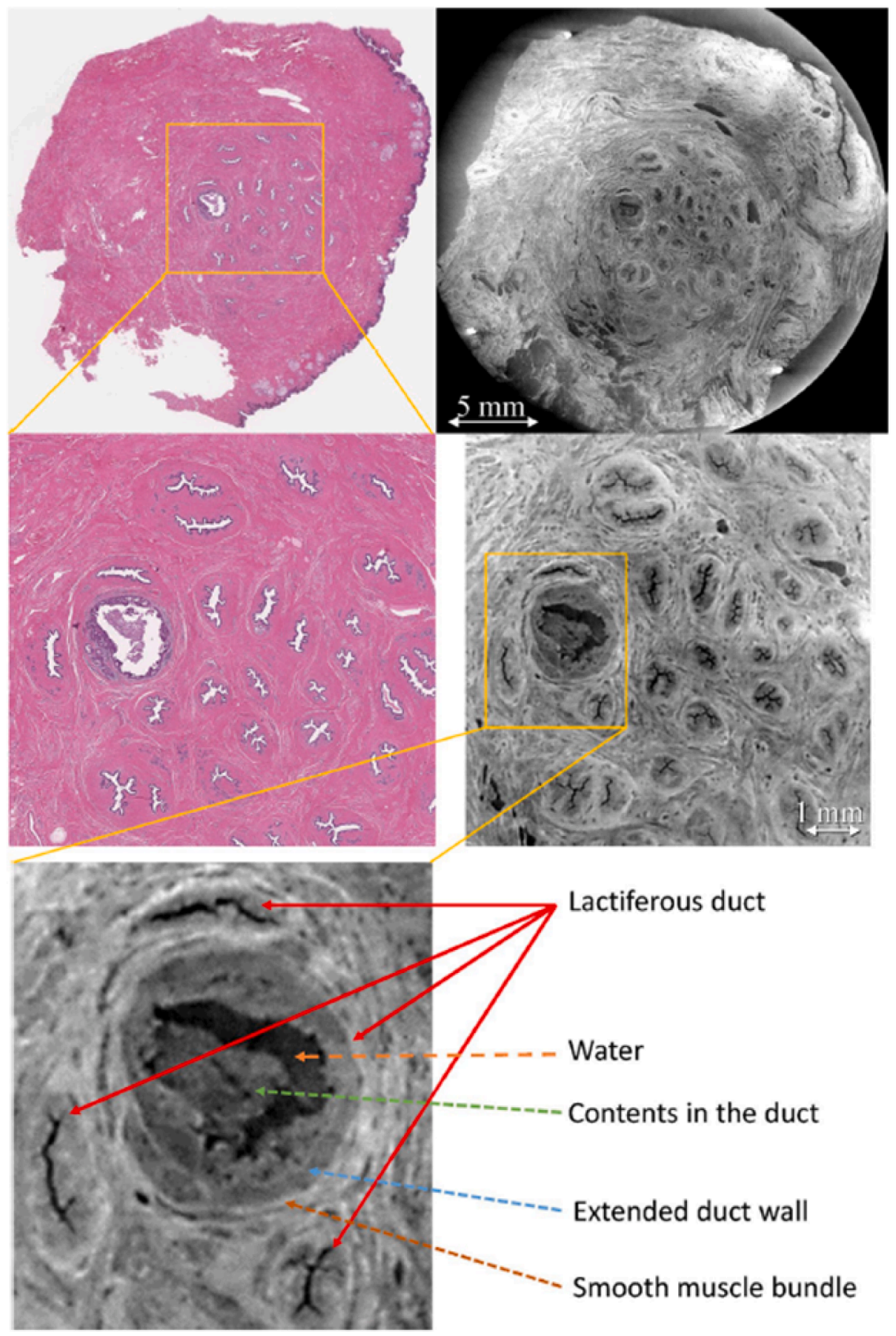

Fig. 14. Visualization of lactiferous ducts in a nipple. The left top shows a histological section while the right top shows section of XDFI-CT image at the same place as the left. The second row shows enlarged view of the top views. The third row shows further enlarged one of the second X-ray view.

microscopic images of stained histological sections without the need for a dye or contrast agent [95-99]. Preliminary phase-contrast studies have focused on the potential of this technology to improve the ability to detect subtle indications of malignant neoplasms, such as spiculations and structural distortions, to identify breast cancer via mammography. Pisano et al. conducted a pilot study to compare DEI with digital radiography using conventional X-rays in formalin-fixed human invasive breast cancer specimens. Histologic whole-mount slides were used as references [95]. Each specimen was approximately $1 \mathrm{~cm}$ thick and sealed in a plastic bag compressed between two Lucite plates. DEI provided better visualization of the lesion spiculation and structural distortion in the breast cancer specimens in six of the seven cases. In these preliminary studies, however, it was not clear whether the terminal duct lobular unit (TDLU) and ducts could be identified by phasecontrast X-ray imaging.

\subsection{Terminal duct lobular unit (TDLU)}

Using XDFI, Ando et al. reported that phase-contrast X-ray imaging enables the visualization of terminal duct lobular units (TDLUs) and the corresponding ducts, which are fundamental anatomical structures of the mammary glands $[91,100]$. These two basic microscopic structures are embedded within fibro-adipose tissue, so they are difficult to discern by conventional mammography without a contrast agent. Both 


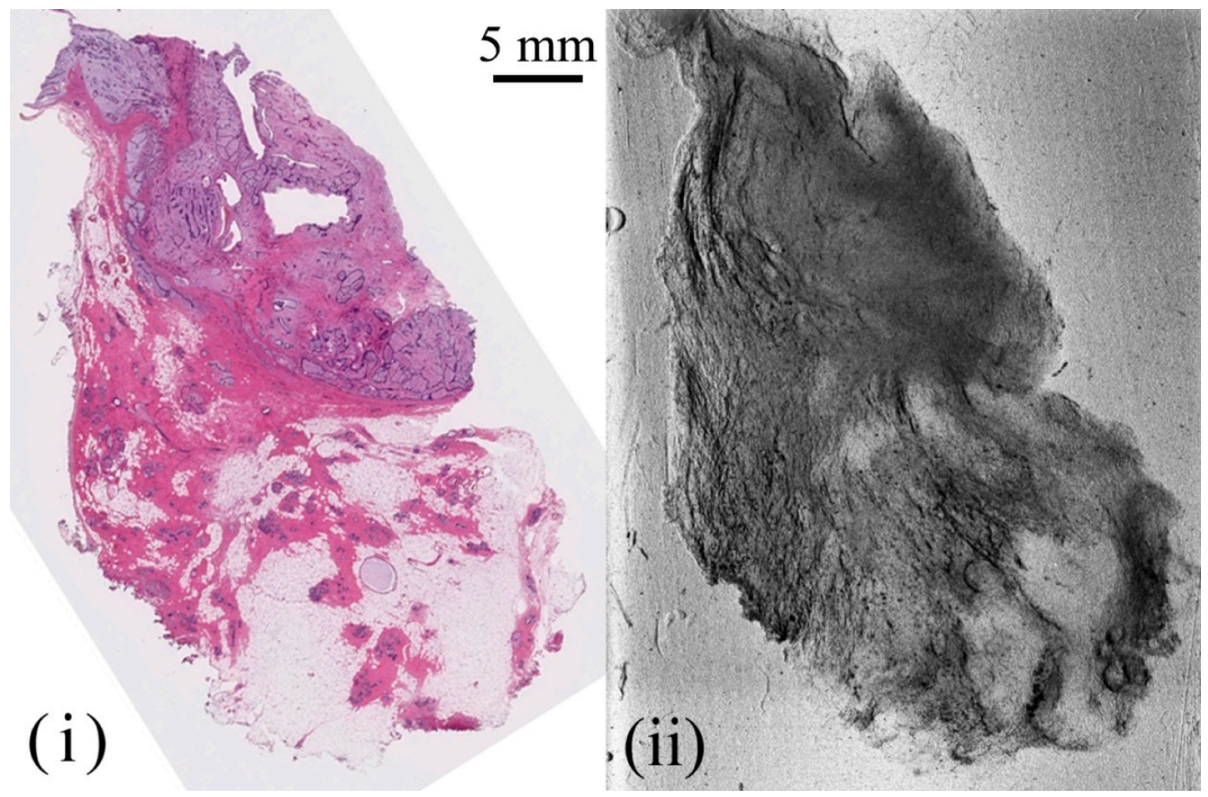

Fig. 15. (i) HE-stained section and (ii) XDFI image of a benign phyllodes tumor from a 37-year-old woman with palpable stiffness in her left breast [100].

structures are generally visualized on stained histological sections, but distinguishing between the TDLU and the ducts is difficult because histological sections obscure the 3D relationship between them. In that study, XDFI was used to visualize a normal TDLU as a tiny refractive structure in a thick section and nodular adenosis in an enlarged TDLU resulting from acinus proliferation, which appeared larger and more refractive than a normal TDLU. The XDFI images of the TDLUs were consistent with those of HE-stained thick tissue sections observed by stereomicroscopy (Fig. 13). Although the sample size was too small to generalize these findings, they strongly suggest that XDFI allows the visualization of the TDLU and ducts without a contrast agent or dye.

\subsection{Microanatomy of the nipple}

Sunaguchi et al. used XDFI-CT to explore the anatomy of the human nipple [101]. In this study, 1428 nipple ducts across 51 nipple samples obtained from mastectomy patients who had been diagnosed with breast cancer (DCIS or invasive carcinoma) via core needle biopsy or fineneedle aspiration were imaged. The images showed the 3D arrangement of the main ducts within the nipple (Fig. 14,). The average number of lactiferous ducts was 28 (the standard deviation was 9.1, and the median was 27).

These results agreed with those from previous studies using conventional methods $[102,103]$. The ostium (just beneath the tip of the nipple) was shared in 525 of $1428(36.8 \%)$ lactiferous ducts. The average number of ostia in which two or more ducts merged was 3.6. Except for the common ostium just beneath the tip of the nipple, there was no anastomosis between the lactiferous ducts within the nipple, which is consistent with the sick-lobe theory proposed by Tan and Tot [104]. Without XDFI, the 3D study of such large samples would not be feasible.

\subsection{Benign and malignant breast tumors}

\subsubsection{Adenosis}

Diagnosing adenosis can be challenging for pathologists because it occasionally mimics invasive cancer under 2D observation, particularly when it is associated with sclerosis. However, adenosis and invasive carcinoma can be readily distinguished from XDFI images: adenosis appears more refractive than invasive carcinoma, which appears less refractive and more homogeneous than normal TDLU. More (a)
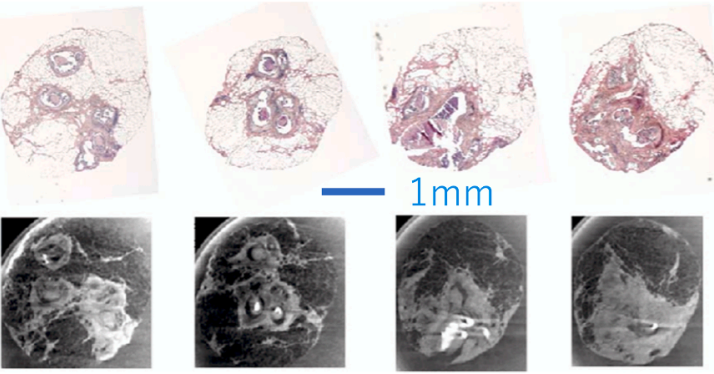

$1 \mathrm{~mm}$
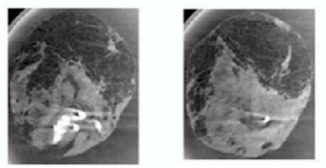

(b)

3-D Reconstruction of casting type calcifications

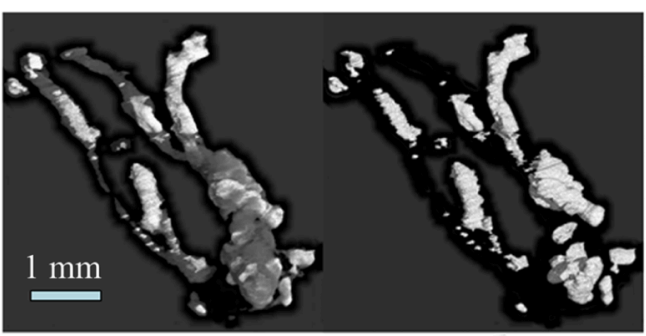

(i)

(ii)

Fig. 16. (a) Four representative images obtained using DEI-CT (lower row) and the corresponding histological HE-stained sections (upper row) [91]. (b) Reconstructed 3D images of DCIS from 900 serial 2D images obtained using DEI-CT. (i) Reconstructed DCIS showing calcification (white) and a noncalcified tumor (gray). (ii) Reconstructed DCIS showing only calcification (white). The pathognomonic linear and branching-type microcalcifications (white) developed within the necrotic zone of the continuous DCIS (gray) [91].

importantly, adenosis is associated with an exaggerated elongation of the ductal structures in the overall 3D lobular ductal arrangement. Because 3D interpretation of such elongation is impossible with light microscopy, XDFI-CT may enable better diagnoses by providing a better $3 \mathrm{D}$ perspective of the anatomy than conventional 2D histopathology.

\subsubsection{Benign phyllodes tumor}

Benign phyllodes tumors and fibroadenoma rarely infiltrate the surrounding healthy breast tissue, instead displacing it in most case. 


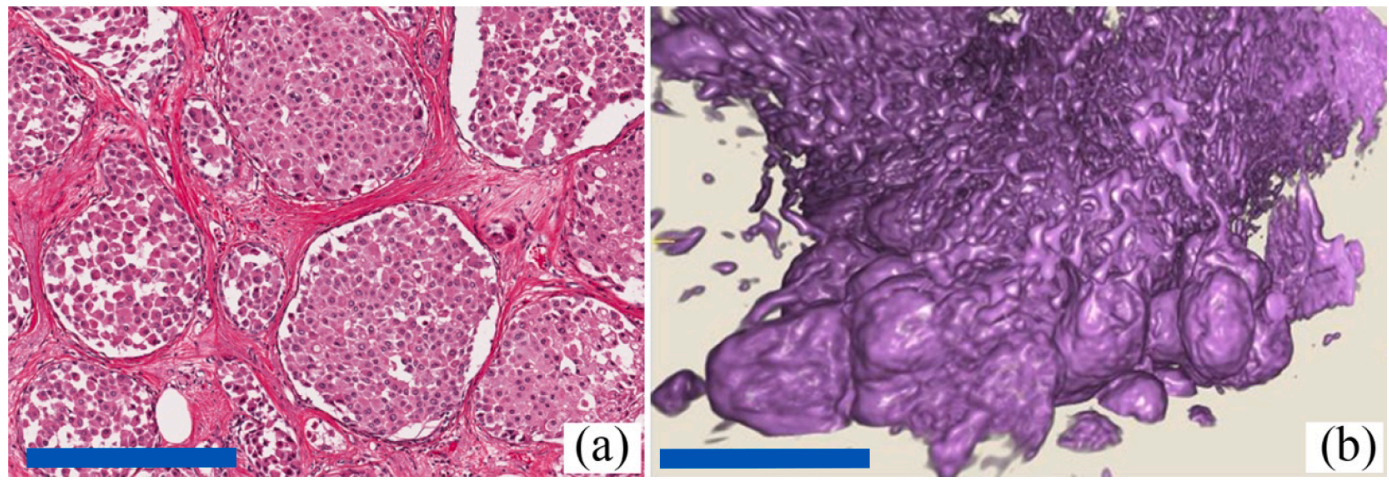

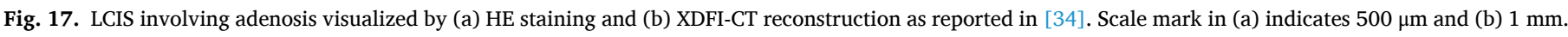

Fig. 15(ii) shows an XDFI image of a benign phyllodes tumor [100]; this tumor was a well-circumscribed homogeneous mass that compresses the surrounding normal breast parenchyma. XDFI made it easy to determine whether the tumor growth was expansive or infiltrative, because the normal structures, including the TDLU, ducts, and vasculature, were clearly visible within the surrounding normal tissue.

\subsubsection{High-grade ductal carcinoma in situ (DCIS)}

We previously visualized two high-grade DCISs with comedo patterns. Conventional mammography showed heterogeneously dense breast parenchyma with suspicious microcalcifications, and the absorption image was equivocal because the soft-tissue contrast was poor. However, the phase image obtained by XDFI revealed dilated, epithelium-lined structures containing hyperintense material, which clearly indicated the presence of a DCIS as shown in Fig. 16. The diagnosis was confirmed by histopathologic observation [100].

To demonstrate the ability to elucidate pathological sites of calcifications, a mammary DCIS was reconstructed from the refraction-based CT images and observed virtually. The specimen was an extensive DCIS with comedo-type necrosis extracted by mastectomy. The 3D reconstruction using DEI revealed that the linear and branching-type microcalcifications observed on the mammograms resulted from multifocal calcifications that developed within the necrotic foci of the continuous DCIS (Fig. 16b) [91].

\subsubsection{Pleomorphic lobular carcinoma in situ (LCIS) involving adenosis}

XDFI provides high-contrast images of internal structures, allowing for malignant tumors to be distinguished from benign ones to some extent. To demonstrate this capability, we visualized and characterized an uncommon morphologic pattern of lobular neoplasia: LCIS involving adenosis. This is a preinvasive condition that can occur in areas of adenosis [105]. The growth of lobular neoplasia in breast tissue that is altered by sclerosing adenosis gives rise to unusual patterns that might be mistaken as invasive carcinoma or DCIS. Distinguishing between these conditions is clinically important because they are treated differently.

Differential diagnosis requires the identification of lobulocentric patterns by histopathological examination and lack of cohesiveness of the component cells. To confirm this condition, immunohistochemical studies for E-cadherin and myoepithelial markers such as p63 and calponin are necessary.

3D reconstruction based on XDFI-CT data revealed that the 3D configuration of the region with adenosis with LCIS looks like tuberous plants such as potatoes (Fig. 17).

In Fig. 17 is shown LCIS involving adenosis visualized at (a) HE staining and (b) XDFI-CT reconstruction [34]. Since the current spatial resolution of $8.5 \mu \mathrm{m}$ is not high enough to be able to visualize inner adenosis.

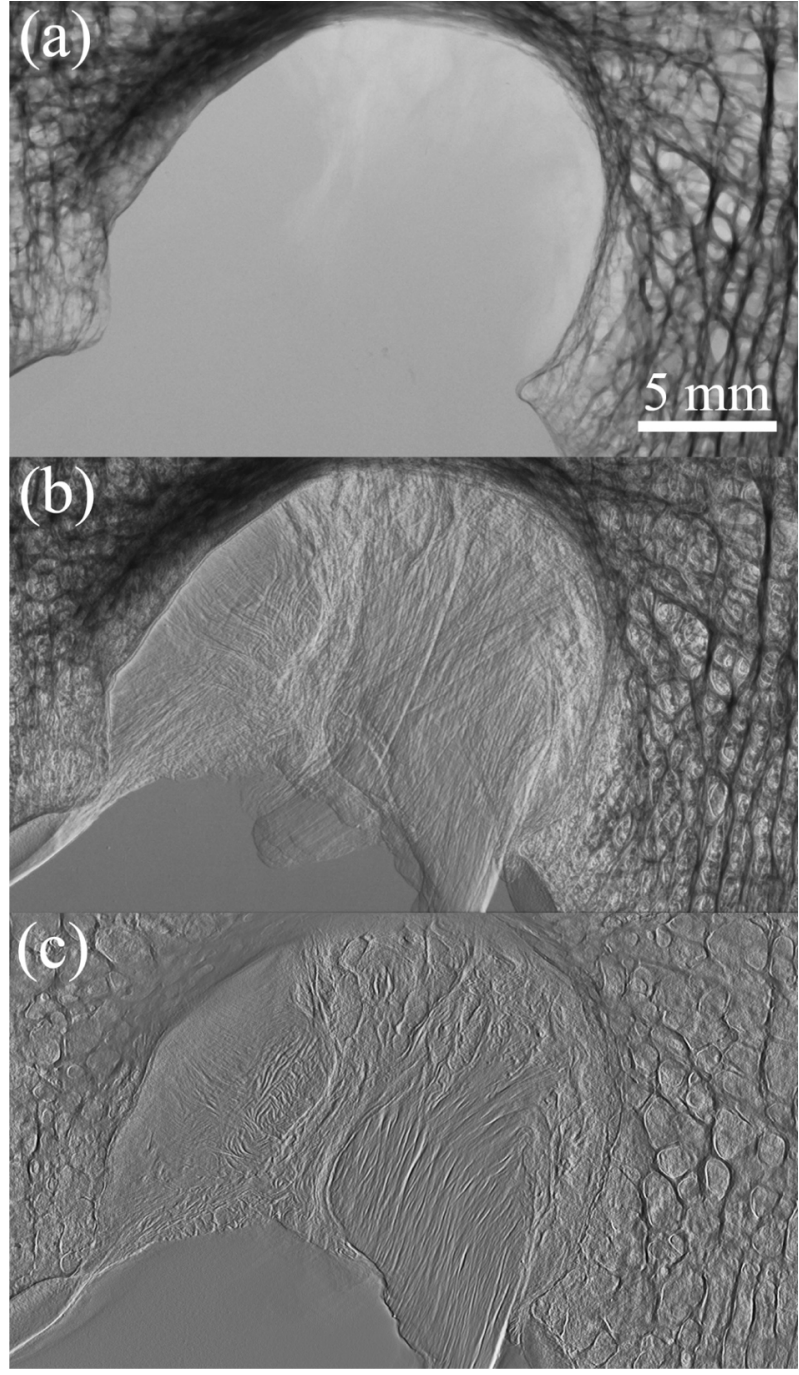

Fig. 18. Intercondylar fossa visualized by (a) absorption contrast X-ray imaging, (b) XDFI, and (c) XDFI-tomosynthesis.

\subsection{Invasive carcinoma:}

Invasive carcinomas can also be visualized by XDFI: they appear as poorly defined, relatively homogeneous masses with less refraction than normal TDLU. Unlike the phyllodes tumor presented above, the normal TDLU and ducts of the adjacent breast tissue are infiltrated by tumor tissue. Recently some researchers have advocaed for using micro X-ray 


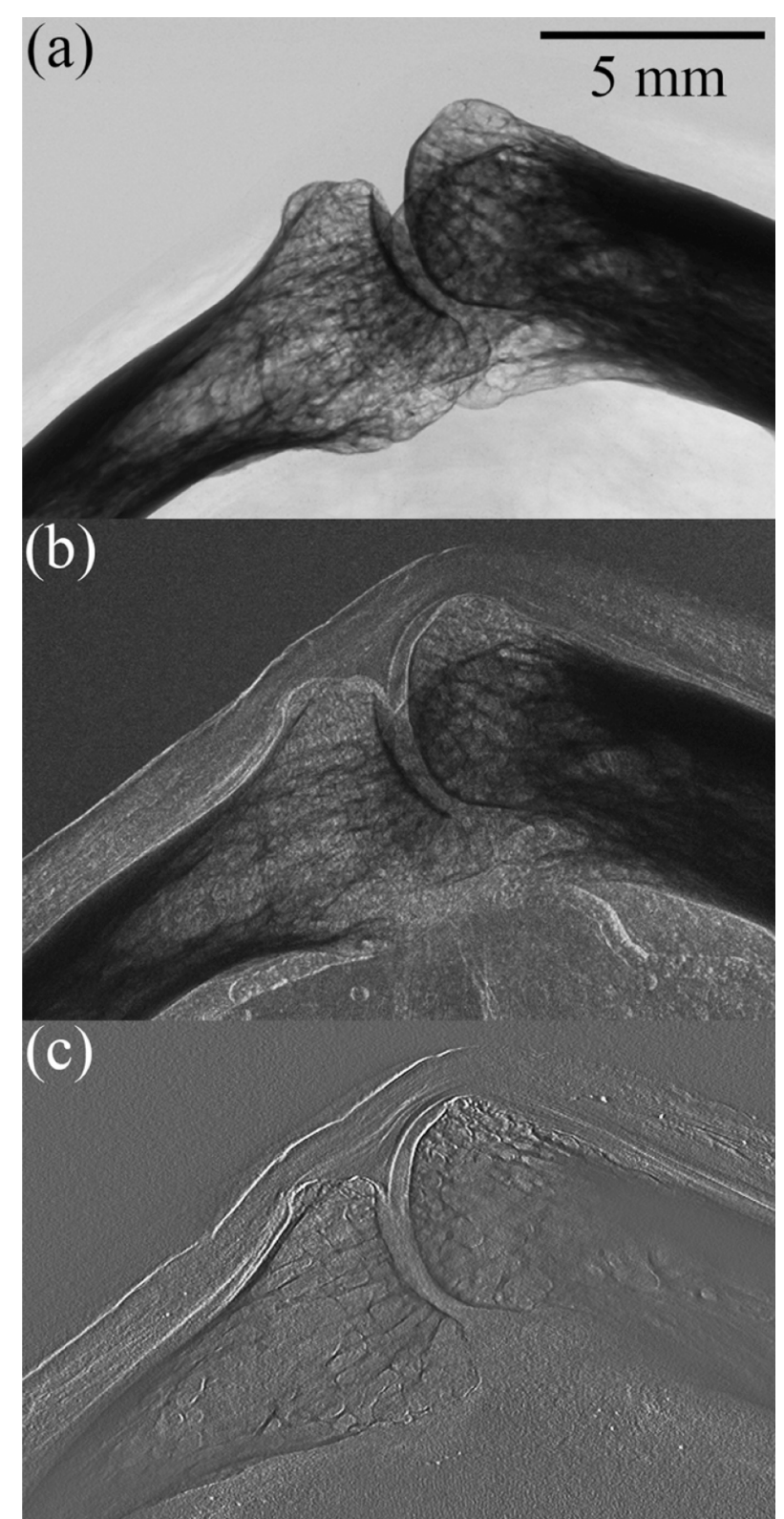

Fig. 19. Finger joint visualized by (a) absorption-contrast X-ray imaging, (b) XDFI, and (c) XDFI-tomosynthesis, clearly showing the extensor digitorum tendon.

CT as a vertual histological tool with or without subsequent classical histology [106].

\section{Clinical approach}

\subsection{Attempt at application to orthopedics}

In orthopedics, CT generally provides more information for diagnostics than simple projection radiography because it offers high resolution along the depth direction. However, projection images from multiple directions (usually over about $180^{\circ}$ ) must be collected for CT, resulting in a longer imaging time, higher radiation exposure, and greater burden on the patient. Furthermore, in the XDFI methods proposed here, synchrotron X-rays are currently required, so it is necessary to rotate the subject to change the projection direction. These limitations pose a high barrier to widespread clinical implementation of CT. Therefore, we investigated the use of tomosynthesis [107] to obtain tomographic XDFI images of arbitrary depth from projected XDFI images obtained within a limited angle range (XDFI-tomosynthesis) $[108,109]$.

First, a human distal femur sliced in coronal sections with $5 \mathrm{~mm}$ thickness through the intercondylar fossa [110] was used to evaluate the feasibility of XDFI-tomosynthesis. Projected XDFI data was collected at BL14B in PF, KEK with an X-ray energy of $20.0 \mathrm{keV}$ using a Si(111) double-crystal monochromator in the optical hutch. The thickness of the LAA ( $\mathrm{Si}(220)$ ) was $0.565 \mathrm{~mm}$. The sample was rotated $\pm 25^{\circ}$ in increment of $1^{\circ}$; thus, 51 projection images were obtained. Then, the XDFI-tomosynthesis images were reconstructed by filtered backprojection using a Shepp-Logan filter.

Absorption-contrast and XDFI projection images of the intercondylar fossa are shown in Fig. 18 (a) and (b), respectively. Only the trabecular structure of medial and lateral condyles can be seen in the absorption image (Fig. 18(a)). The XDFI image additionally shows the posterior cruciate ligament and articular cartilage on the condyles (Fig. 18(b)), demonstrating the ability to attain excellent visualization of soft tissue using XDFI. Furthermore, the XDFI-tomosynthesis image at half-depth (Fig. 18(c)) shows higher resolution in the thickness direction compared with the XDFI image, and the trabecular structure and running fibers of the ligament can be clearly observed. This experiment with a sliced specimen preliminarily demonstrated that XDFItomosynthesis is possible.

As the next step, the potential for XDFI-tomosynthesis was verified on an intact specimen that was not sliced. A formalin-fixed cadaveric human finger (the third digit) $[111,112]$ as the specimen. The X-ray optics, imaging conditions, and XDFI-tomosynthesis reconstruction algorithm used were the same as those used for the human distal femur. The absorption-contrast and XDFI projection images of the proximal interphalangeal (PIP) joint of the finger obtained from a lateral oblique view are shown in Fig. 19(a) and (b), respectively [34]. Only the cortical bone and trabecular structure can be seen in the absorption-contrast image (Fig. 19(a)).

However, the XDFI image additionally shows the articular cartilage on the head of proximal phalanx and the extensor digitorum tendon (Fig. 19(b)), further demonstrating excellent visualization of soft tissue by XDFI. The XDFI-tomosynthesis image of the PIP joint at half-depth (Fig. 19(c)) clearly shows the articular cartilage, tendon, and trabecular bone structure at the focus depth. This finding further supports the potential for using XDFI-tomosynthesis to image intact subjects.

Compared with XDFI-CT, XDFI-tomosynthesis can reduce the number of projections, the exposure dose, and the physical burden on the patient arising from rotational movement. Although the image quality with XDFI-tomosynthesis is not nearly as high as that with XDFI-CT reconstructed by projection images from a $180^{\circ}$ range, it is more compatible with practical clinical use.

\subsection{Neuroimaging}

Many imaging modalities including CT, MRI and PET are available for neuroimaging. For any particular indication such as trauma or stroke, one can define the value of the imaging modality based on experience and practice-base criteria that categorize the modality as defined below.

- Level 1: Nearly always valuable for patient care, and successfully obtainable in the vast majority of patients.

- Level 2: May be valuable for patient care, and is successfully obtainable in most patients

- Level 3: Valuable for research purposes and may or may not help in the management of the patient.

One could also use an evidence base classification for recommendation, define as follows. 

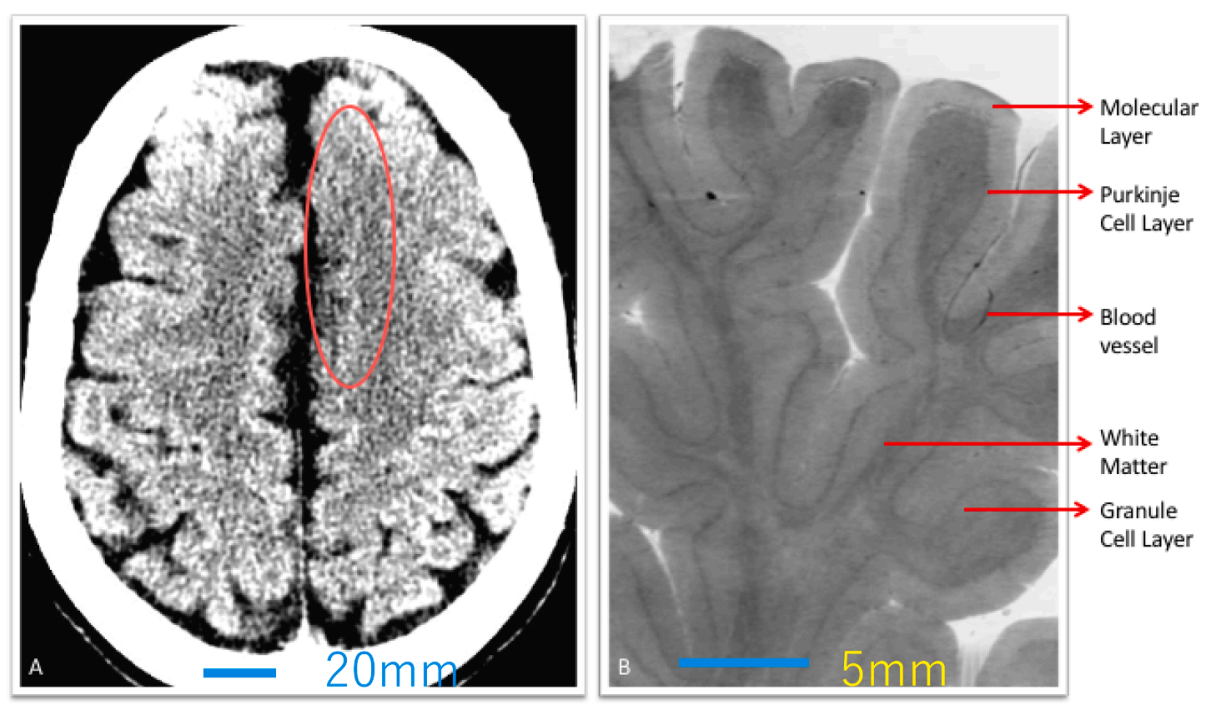

Fig. 20. A non-contrast head $\mathrm{CT}$ in a patient with acute ischemic stroke in the left anterior cerebral territory as demonstrated by the subtle loss of the cortical ribbon (red ellipse) reflecting loss of gray-white differentiation because of acute ischemia. The right-side panel shows XDFI image of a cerebellum demonstrating gray-white differentiation that surpasses a typical MRI scan.

- Class I: An imaging modality for which there is evidence for and/or general agreement that it is beneficial, useful, and effective in management of acute stroke.

- Class II: An imaging modality for which there is conflicting evidence and/or divergence of opinion about the usefulness/efficacy in management of acute stroke.

o Class IIa: Weight of evidence or opinion is in favor of usefulness or efficacy of this imaging modality.

o Class IIb: The usefulness or efficacy is less well established by the evidence or expert opinion.

- Class III: An imaging test for which there is evidence and/or general agreement that it is not useful or effective, and in some cases may be harmful, in the management of acute stroke patients.

These categories are only suitable for describing efficacy of an imaging test when it is clinically available. From this perspective, XDFI is still too experimental to classify on the above metrics. Nonetheless, XDFI demonstrates capabilities and features that could augment and improve imaging modalities that heretofore have been considered Level 1/Class 1 indications for a particular clinical presentation.
Take the case non-contrast CT (NCCT) scan of the head in the management and triage of acute stroke in Emergency Department. There is broad agreement that NCCT is a Level 1/Class I test in the management of acute stroke. It is widely regarded as repeatable and reliable. While not $100 \%$, there is Level 1 evidence documenting is efficacy in excluding intracranial hemorrhage and mass lesions. There is also Level 1/Class I evidence that NCCT can identify a subset of acute strokes directly: nearly three-quarters of large stokes can be identified in the first $6 \mathrm{~h}$ on NCCT images. Decision to administer thrombolytic treatments such as intravenous (IV) tissue plasminogen activator (tPA) based on NCCT has Level $1 /$ Class I support as it has been established by multiple large randomized clinical trials. In fact, NCCT is often the only study done before administering IV tPA.

Nonetheless, NCCT has many limitations that can be improved or ameliorated with the help of XDFI. For example, assessment of stroke volume on NCCT, a key determinant of whether a patient is suitable for intra-arterial therapy such as catheter-assisted thrombectomy, only receives a score of Level 2/Class IIa. The Alberta Stroke Program Early CT score (ASPECTS), a 10-point quantitative topographic CT scan score is widely used to score the volume of infarcted territory. ASPECTS is
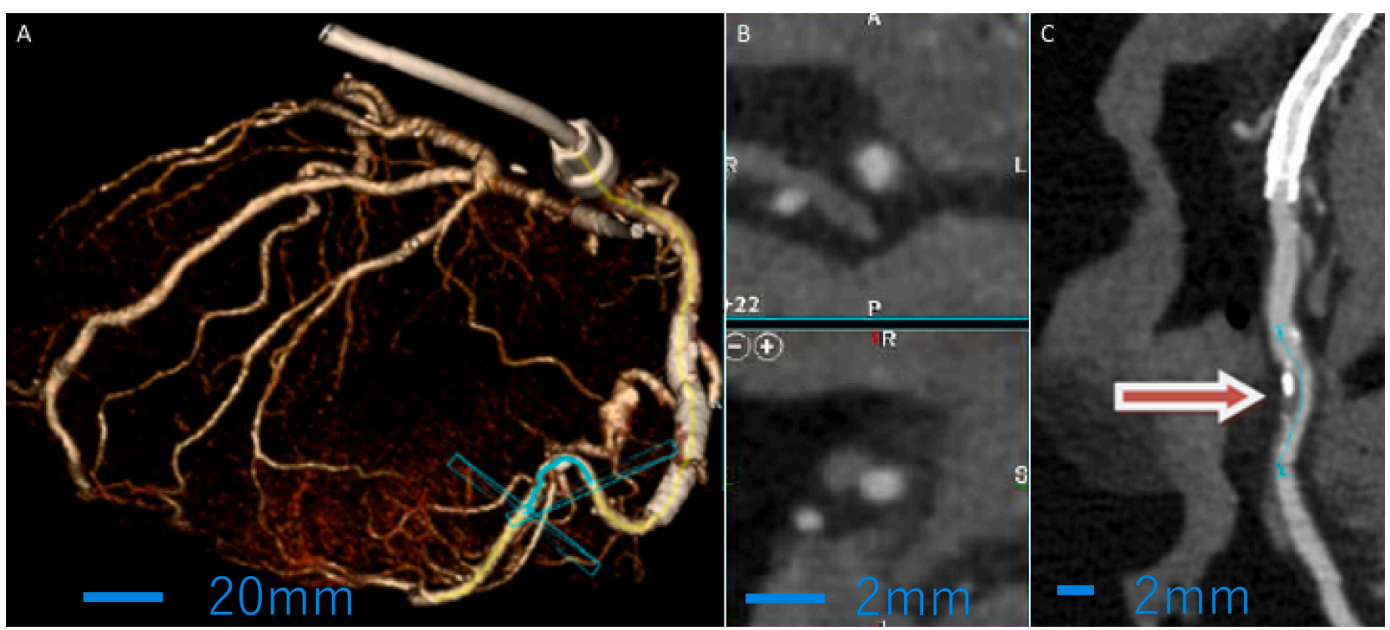

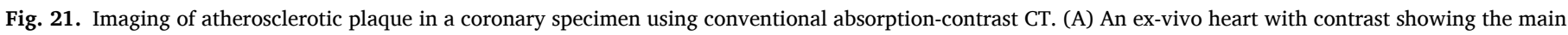

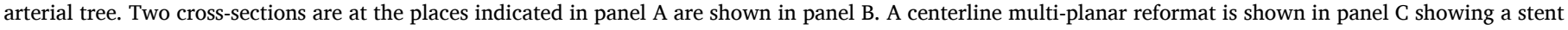
and a segment with moderate to severe stenosis (arrow). 


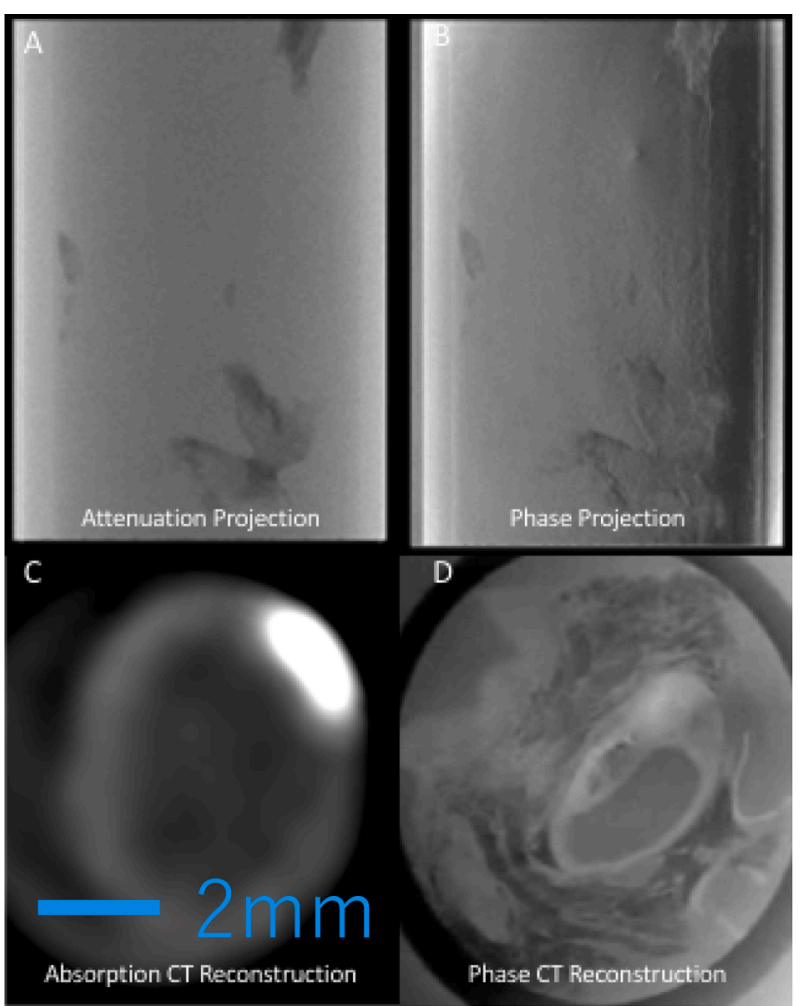

Fig. 22. Phase projection images of an artery with extensive atherosclerotic disease (top panels A and B). Panel C shows a routine, conventional absorption contrast CT scan through a diseased artery. A phase-contrast tomographic section is shown Panel D. Notice the significant increase in the contrast resolution and soft tissue contrast between absorption and phase contrast CT slices.

thought to offer a reliable and repeatable scoring system to assess early ischemic changes on pre-treatment CT studies. However, determination whether a particular region is infarcted or not can be very unreliable on a non-contrast CT, especially early in the course of the stoke onset.

Fig. 20 shows the case of an early ischemic stroke in the left anterior cerebral artery (Panel A). As can be seen, the infarcted tissue has subtle loss of gray-white differentiation. In general, a CT scan performed very early in the evolution of an ischemic stroke has limited value in showing the infarcted area. Its main value is in excluding other causes of neurological worsening and in ruling out intracranial hemorrhage. The improved soft tissue contrast of XDFI as can be seen in Fig. 20(B) can not only show the gray and the white matter, it can show multiple intervening layers in the organization of the brain parenchyma that are not visible at conventional CT.

Most cases of acute ischemic stroke arise from emboli originating from carotid arteries with severe atherosclerotic disease. In order to evaluate the difference between phase and absorption contrast images of atherosclerosis, we applied XDFI and conventional CT to a heavily diseased artery containing atherosclerotic plaque [113].

Fig. 21 shows the absorption images of an ex-vivo heart specimen where the arteries have been injected with iodinated contrast. The conventional CT images show only the calcified portion of this atherosclerotic plaque. Despite extensive disease in this arterial specimen, the absorption tomographic slices fail to show the morphology of the plaque.

XDFI has superior contrast over absorption for soft tissue processes such as atherosclerotic plaque [113], as shown in Fig. 22 which directly compares the absorption and phase images both in the projection domain as well as tomographic domain. While the absorption images only show the lumen and calcifications, the phase images provide internal morphologic details of the atherosclerotic plaque components. For example, the structure of the vessel wall consisting of the adventitia,

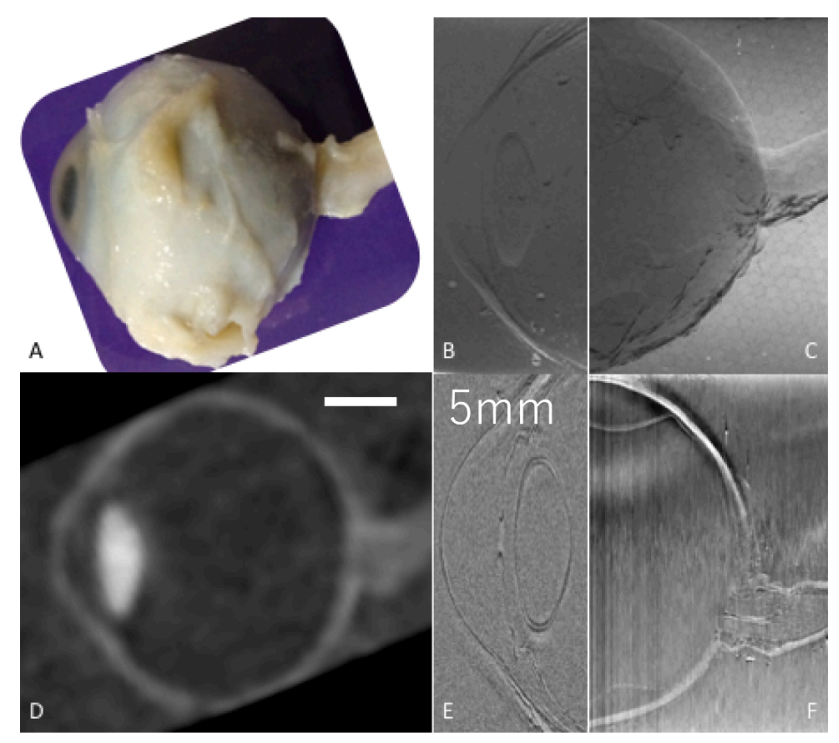

Fig. 23. (A) An ex-vivo eye specimen showing the globe and a short segment of the optic nerve; (B) and (C) show XDFI projection images through the anterior and posterior segment of the specimen in A; Panel D shows a conventional CT scan of the specimen while Panel E and F show the phase contrast XDFI images through the anterior and posterior chambers.

the media and the intima can be appreciated. One can also visualize various components such as the fibrous cap and the atheroma within the plaque. These structures are distinct from the calcification which shows artefacts from a phase saturation effects. These images clearly establish the complementary nature of XDFI as it adds details that are invisible in absorption images.

The superior soft tissue contrast obtained with phase contrast imaging in both 2D projection and 3D tomographic domains can also be leveraged in other pre-dominantly soft tissue organs such the eye. In order to illustrate this difference, we imaged an enucleated human eye specimen using both XDFI and conventional CT imaging [34,86,113].

Fig. 23(A) shows an image of the specimen consisting of an intact globe along with a short segment of optic nerve. Because the entire specimen did not fit inside the field of view of our XDFI setup, the anterior and posterior chambers of the eyeball were imaged separately. Fig. 23(B) and (C) show sagittal projections of the differential phase map of the anterior and posterior chambers respectively. The absorption image from conventional CT is shown in Fig. 23(D). The absorption image only shows the lens and the broad outline of the soft tissue anatomy of the globe. The reconstructed phase images (Fig. 23(E) and (F)) are able to delineate multiple structures within the globe. The markedly increased detail and contrast, especially at the interfaces, is quite obvious in the XDFI images which clearly demonstrate the margins of the cornea, iris, pupil, ciliary body, angle, lens, and the suspensory ligament of the lens. The images of the posterior globe and the site of optic nerve insertion of the specimen clearly shows the tri-laminar structure of the globe consisting of the retina, the choroid and the sclera. The insertion of the optic nerve into the globe is clearly seen along with the central retinal artery, the nerve fascicles and the optic nerve sheath which is contiguous with the sclera.

The XDFI section through the eye globe also shows separation of the retinal and choroidal layers from the underlying sclera at multiple places, most likely due to the post-mortem condition, prolonged formalinfixation, and contraction of the vitreous humor (Fig. 23). Irrespective of the cause, the retinal and choroidal detachments are well demonstrated by XDFI. In fact, the phase contrast image can show the location of the fluid to be in the sub-retinal or sub-choroidal location. 


\section{Discussion and future perspective}

\subsection{Development of high-performance X-ray camera}

Over the past 20 years of developing XDFI optics, we have encountered and resolved numerous issues, such as the coexistence of soft and hard tissue components, the asymmetric shape of the left and right rocking curves (which has relation to angular analysis), and spatial resolution to be improved.

Currently, a remaining challenge is to attain higher spatial resolution comparable to that of established standard pathology techniques (i.e., 2-3 $\mu \mathrm{m}$ under an optical microscope when analyzing soft tissue). At present, a commercially available X-ray CCD cameras with a model XFDI 1.00:1 was used. This has a 4878 (horizontal) by 3247 (vertical) matrix of $7.4 \mu \mathrm{m}$ by $7.4 \mu \mathrm{m}$ pixel size, providing with a FOV of $36.0 \mathrm{~mm}$ (horizontal) by $24.0 \mathrm{~mm}$ (vertical). This has been widely used for a long time.

However, to meet the requirements for pathological examination by $\mathrm{XDFI}$, a higher-performance X-ray camera is needed. This is a fundamental hurdle that must be overcome to advance this technology in the field of pathology.

\subsection{Potential for visualizing brain tumors through the skull : Mitigating contrast loss in X-ray diffraction transcranial brain imaging}

We have been trying to use XDFI to visualize many human organs except the brain, which is uniquely challenging to image because it is surrounded by the skull. The skull comprises compact bone and cancellous bone, which has a sponge-like appearance with numerous microscale $(200-400 \mu \mathrm{m})$ pore-like spaces . In our study of transcranial brain imaging [114] using DEI-CT, we found that this structure created a technical issue: DEI X-rays pass through the specimen, they are supposed to be detected by a BAA, which has a symmetric rocking curve height along the $\mathrm{y}$-axis and a refraction angle along the $\mathrm{x}$-axis. Because each reflectance value corresponds to two different refraction angles, the angle should be unambiguously determined by the appropriate algorithms in the reconstruction software to prevent mismatching of angles. Additionally, when the refraction angle is beyond the measurable limits imposed by the analyzer rocking curve, an arbitrary value can be assigned in the conventional XDFI-CT reconstruction software. In our wave-propagation simulation and sinogram analysis of XDFI-CT on a real skull-brain phantom, we found that X-ray refraction and scattering by the skull micropores affect the measurement of sample-induced refraction angles in the LAA in two ways, causing both left-right angle mismatches and out-of-range mismatches. These effects impacted the curve-fitting of the refraction angles with the analyzer rocking curve and reduced the contrast in the intracranial structures. However, we demonstrated that the inner structures could be recovered by correcting these angle mismatches or artificially widening the rocking curve to cover a broader range of X-ray energies.

For clinical applications, it is desirable to resolve the contrast loss arising from the scattering effects associated with the micropores in the skull. This can be achieved by designing a novel LAA with a wide rocking curve width.

\subsection{Extraction of SAXS contrast from XDFI-CT images}

Alternatively, the contribution of small-angle X-ray scattering (SAXS) could be extracted by acquiring pure absorption-based CT data subtracting the absorption contrast from the XDFI-CT data. This approach is expected to be effective because microporous structures may not produce SAXS if the critical diameter of scattering $(20 \mu \mathrm{m})$ is smaller than the pore size of the skull $(100-400 \mu \mathrm{m})$. Thus, SAXS contrast could be separated from refraction-based contrast in the XDFI-CT image. However, internal structure-mediated SAXS-related contrast may arise from factors other than the interference from the microporous skull, such as a higher sensitivity to angular deviation compared with the refraction-based imaging.

\subsection{Towards more robust X-ray optics}

Based on the relationship between the detection range and the width of the rocking curve, there may be a way to alleviate the contrast loss in transcranial XDFI-CT, such as that which occurs in brain imaging, by designing a new analyzer. We conducted a wave-propagation simulation to demonstrate that using an analyzer crystal with a double-width rocking curve results in a $12 \%$ reduction in the out-of-range mismatches in the detection of refraction angle-based reflectance; this resulted in the restoration of contrast in the internal structure. This finding suggests that designing an analyzer with a wider rocking curve may resolve the contrast loss in analyzer-based X-ray transcranial imaging, which is expected to improve the visualization of inner structures upon post-image processing. It is technically possible to fabricate an analyzer crystal with a wider rocking curve by using materials with low refractive indices, such as germanium, or composite crystals.

However, it will take a long time to develop highly perfect, largesize, high-Z crystals. Unfortunately, currently available germanium crystals and composite crystals, such as GaAs crystals, are poor quality: for instance, the number of dislocations (which is a common metric for crystal quality) usually exceeds $1000 / \mathrm{mm}^{2}$.

\subsection{Large FOV for clinical implementation}

For XDFI to be useful for clinical applications, the optics will need to be developed to attain a large FOV. This could be achieved by introducing a Si single crystal with a diameter of $450 \mathrm{~mm}$, which would increase the FOV to $318 \mathrm{~mm}$ by $318 \mathrm{~mm}$; this FOV would be suitable for a variety of applications including mammography. However, to test this modification, a beamline exceeding $100 \mathrm{~m}$ in length will be needed to obtain a large FOV.

\subsection{Need for a compact radiation source}

The development of more compact radiation sources would accelerate the clinical implementation of XDFI at facilities all over the world. A few designs have been proposed, including one [115] using laserCompton light source technology. However, there is currently no decisive candidate for such a radiation source.

\subsection{Development of XDFI optics for higher-energy X-rays}

Most of our studies were conducted using an X-ray energy of $35 \mathrm{keV}$ with the BL14C and $20 \mathrm{keV}$ with the BL14B. Both of these beamlines use the same radiation source, a 5 Tesla superconducting wiggler, which produces a spectrum with a critical photon energy of $20 \mathrm{keV}$. This X-ray energy is not high enough for more advanced applications, such as visualizing a brain tumor through the skull. This issue could be addressed by using a radiation source producing higher-energy X-ray photons. At the same time, novel optical components for the XDFI system, especially the AMC, would be needed. Alternatively, this issue could be mitigated by developing a new algorithm to suppress the unwanted phase distortion each time the X-rays pass through the skull. 
Table 2

Comparison of scan parameters between PBI and XDFI.

\begin{tabular}{|c|c|c|c|c|c|c|c|c|c|}
\hline & specimen & $\mathrm{X}$-ray energy keV & polarization & FOV $\mathrm{mm}^{\mathrm{H}} \approx \mathrm{mm}^{\mathrm{V}}$ & voxel & Dose rate & frame & Exposure time second & Rad dose kGy \\
\hline \multirow[t]{2}{*}{ PBI } & mouse & 22 & horizontal & $4 \sim 5.2$ & $2-2.6$ & High & 1800 & 2 & 80 \\
\hline & & 19 & & & & Low & 900 & 0.05 & 2 \\
\hline XDFI & Human organ & 20,35 & vertical & $36 * 24$ & 7.4 & low & $51,360,600,900$ & $1 \sim 10$ & $84 * 10^{-6} \sim 0.01$ \\
\hline
\end{tabular}

\subsection{XDFI should be a tool to coexist genetic analysis}

In recent years, the trend toward precision therapy based on comprehensive genetic analysis is accelerating, and it is becoming more and more practical to perform subsequent genetic testing using clinical specimens from archived cancer patients. In this situation, we believe that the conventional invasive pathological method of preparing specimen by sectioning coupled with 3D X-ray image reconstruction is not practical. Focusing on 3D histological methods together with help of non-invasive imaging by XDFI can be expected as an alternative to the classical pathology. In that case, pathology diagnosis by XDFI would be practical if it could verify that XDFI will not affect quality of the genes in the specimen by $\mathrm{X}$-ray radiation dose.

\subsection{Preliminary consideration for radiation dose in case of clinical test}

We have a natural wish to bring this XDFI optics to a clinical mode in a future so that we report here in this note our two trials towards clinical application. One is checking the status of bone joints of finger and knee taken almost similar to a clinical condition in the Section 9.1 and the other is checking coronary status in 9.2. From a clinical view application of XDFI to orthopedics seems promising due to two reasons. One is possibly being able to see internal structure with layer by layer in pseudo $3 \mathrm{D}$ view by tomosynthesis that can reduce significantly the radiation dose. As a next step clinical application to breast cancer seems promising. Already we measured the expecting skin radiation dose of breast check under the XDFI optics in 2011 [116]. Also we would like to consider another possibility of viewing coronary arteries in a clinical mode. However we may need further steps to reach this at clinical level such as expanding FOV, further development of XDFI optics adaptive to higher X-ray energy etc. In a nearest future if these conditions will be satisfied clinical estimation of radiation dose will be available. At the same time one will need evaluate radiation dose using a usable imaging device that might have higher spatial resolution and higher quantum detection efficiency.

\subsection{Expecting future trend of XDFI in clinical diagnosis}

X-ray dark-field imaging (XDFI) differs from conventional x-ray examinations in that it is possible to make a definitive diagnosis without pathology. This technology could significantly reduce health care time. For example, the mammary gland is scanned with XDFI and the data is uploaded and sent to a medical reader or artificial intelligence somewhere in the world. Then you pay via PayPal or some other means to get the report. Your doctor can then start treatment immediately based on the report.

We have conducted experiments and are approaching clinical trials for visualizing soft tissue in human joints. Furthermore, on the request from, for instance, a breast radiologist, we are investigating the potential to capture whole-breast images, which could ultimately serve as an important clinical trial tool. We intend to leverage future opportunities to utilize synchrotron labs to test these two applications.

\section{Summary}

This note describes applications of XDFI to its assistance to pathology and clinical trial. The pathological application covers mostly breast cancer and clinical one covers check of orthopedics irregularity and blood cells.

The XDFI can provide a 3D perspective to $2 \mathrm{D}$ histopathologic evaluation with high contrast $(1000: 1)$ and resolution $(8.5 \mu \mathrm{m})$ without needing dying or contrast agent. The examination of HE-stained breast cancer tissue under a light microscope which is the basis for established standard histopathological diagnostics needs preparation time and 2D images are available so that assistance from XDFI enabling 3D visualization of morphological abnormalities in tissue with the spatial resolution of $10 \mu \mathrm{m}$ or better might be useful to classical pathology if this XDFI can technically go further such as developing a high-resolution X-ray camera and validating it on human specimens.

Histopathological analysis combined with 3D imaging may enhance the study of normal and disease processes, particularly those involving structural changes, such as cancer. Its combination with conventional histopathological examination could add further value by providing additional information and enhancing the pathological-radiological-clinical correlation.

One-to-one correspondence between histopathological views under an optical microscope and sliced X-ray CT views should be investigated to associate the X-ray appearance of established pathology. Such examinations will enable the identification of all types of tissues and their functions with the X-ray view alone.

At the last but not the least it would be valuable to describe about the propagation based imaging (PBI) which has been developing at ELETTRA at Trieste; they have a long and wonderful history of clinical survey of breast cancer [11,117], this is a quite interested project and we wish to participate this program in a future. We can also compare SR $\mu \mathrm{CT}$ technique under development at ELETTRA [118] with XDFI at Photon Factory (PF). X-ray energy around $20 \mathrm{keV}$ is for PBI and $20 \mathrm{keV}$ and 35 $\mathrm{keV}$ is for XDFI at PF. White radiation with a filter is used for PBI, while $\mathrm{XDFI}$ uses monochromatic radiation that may lead to high contrast. In a low dose case of PBI the data acquisition time is extremely short $\sim 45 \mathrm{~s}$. This is amazing and far faster than XDFI that may need $1.5 \mathrm{~h}$ at minimum. FOV is relatively small in PBI such as $10 \mathrm{~mm}$ in square that is required for imaging a mouse in histology, while FOV of XDFI is currently $36 \mathrm{~mm} \times 24 \mathrm{~mm}$ which is requested from pathology for human. This is a biggest difference between PBI and XDI. From a crystal point of view in a future we can foresee a clinical application of XDFI where FOV can be $318 \mathrm{~mm}$ by $318 \mathrm{~mm}$ at maximum, as described in section 10.5. The voxel size of PBI is extremely small $2 \sim 2.6 \mu \mathrm{m}^{3}$. XDFI at present remains at $7.4 \mu \mathrm{m}^{3}$ of voxel. This means XDFI will need further instrumental development of X-ray camera so that higher spatial resolution at first stage $5 \mu \mathrm{m}^{3}$ and at the second stage $2-3 \mu \mathrm{m}^{3}$ will be achievable. For comparison Table 2 is prepared. Biggest difference is type of specimen such that mouse is at PBI and human tissue at XDFI. Mouse needs a very high spatial resolution, while XDFI also needs high resolution so that the current one should be improved in a nearest 
future. The radiation dose of PBI is quite high because of extremely small voxel size, about three orders higher than of XDFI.

\section{Declaration of Competing Interest}

The authors declare that they have no known competing financial interests or personal relationships that could have appeared to influence the work reported in this paper.

\section{Acknowledgements}

The experiments were performed under proposals 2006G212, 2008S2-002, 2008G081, 2010G157, 2012G521, 2012G562, 2014G589, 2015G597, 2016G0625, 2017G697, 2019G597, and 2019G598 at KEK. The authors would like to express their sincere thanks to the beamline managers at BL14B and BL14C, Professors K. Hyodo and K. Hirano, who kindly let us use their CCD camera to acquire X-ray images to reconstruct CT images, and Professor K. Mori of Ibaraki Prefectural University of Health Sciences for providing a knee sample and supporting our experiment to acquire 2D X-ray images at KEK-PF. We appreciate the advice and expertise of MD Dr. Rieko Nishimura, MD Dr. Yoshiko Murakami, MD Dr. Takako Morita, MD Dr. Mikinao Oiwa, and MD Dr. Masaya Suenaga of NMC, MD Professor Suzuko Moritani of Shiga University of Medical Science, MD Professor Tomonori Kawasaki of Saitama Medical University, and MD Professor Atsushi Enomoto of Nagoya University Medical School. Dr. Suenaga pointed out a very crucial point at 11.8 of discussion so that XDFI should be not only a pathological tool handling excised tissue but also a clinical tool, while giving no genetic damage to DNA. Some of the Si crystals for the imaging optics were prepared at the Crystal Optics Processing Laboratory at PF KEK, Tsukuba, Japan with the help of Dr. T. Sasaya from Yamagata University in Professor Yuasa's laboratory, to whom the authors are indebted. Figs. 7 and 8 were drawn by Dr. Yasuo Higashi to whom the authors very much appreciate for his skill. This research was partially supported by Grantsin-Aid for Scientific Research (grant numbers 18206011, 18790900, 21791204, 22591353, 24601011, 26286079, 15H01129, 16K01369, 16K08654, 17K05393 and 18K13765) from the Ministry of Education, Culture, Sports, Science and Technology and the Aichi Science and Technology Foundation. We thank Dr. Stephanie Knowlton from Edanz Group (https://en-author-services.edanzgroup.com/ac) for editing a draft of this manuscript.

\section{Compliance with ethical standards}

Not applicable because this is a review and perspectives on a new imaging technique.

\section{Appendix}

See below for Figure number and publication number in Reference corresponding to each specimen shown in Table A1.

\section{Table A1}

\begin{tabular}{lll}
\hline Specimen & Figure number & Reference \\
\hline TDLU & 13 & {$[102]$} \\
Nipple & 14 & {$[103]$} \\
Benign phyllodes tumor & 15 & {$[102]$} \\
DCIS & 16 & {$[93]$} \\
LCIS & 17 & {$[35]$} \\
Knee & 18 & {$[109-111]$} \\
Finger joint & 19 & {$[112,113]$} \\
Brain & 20 & {$[114]$} \\
Artery & 22 & {$[115]$} \\
Eye ball & 23 & {$[114,115]$} \\
\hline
\end{tabular}

\section{References}

[1] Ando M. and S. Hosoya, 1972. An Attempt at X-Ray Phase-Contrast Microscopy, Proceedings of the 6th International Conference on X-Ray Optics and Microanalysis, University of Tokyo Press ed. by G. Shinoda, K. Kohra and T. Ichinokawa 63-68.

[2] Bonse U, Hart M. An X-Ray interferometer. Appl Phys Lett 1965;6:155-6.

[3] Podurets KM, Somenkov VA, Shilstein SS. Neutron radiography with refraction contrast. J Physics B 1989;156 \& 157:691-3.

[4] Shilstein SS, Podurets KM, Somenkov VA, Manushkin AA. Refractive radiography of biological objects. Surf Investig 1997;12:451-7.

[5] Ingal VN, Beliaevskaya EA. X-ray plane-wave topography observation of the phase contrast from a non-crystalline object. J Phys D Appl Phys 1995;28: 2314-7.

[6] Davis TJ, Gao D, Gureyev TE, Stevenson AW, Wilkins SW. Phase-contrast imaging of weakly absorbing materials using hard X-rays. Nature 1995;373:595-8.

[7] Snigirev A, Snigireva I, Kohn V, Kuznetsov S, Schelokov I. On the possibilities of $\mathrm{X}$-ray phase contrast microimaging by coherent high-energy synchrotron radiation. Rev Sci Instrum 1995;66:5486-92.

[8] Chapman D, Thomlinson W, Johnston R, Washburn D, Pisano E, Gmür N, et al. Diffraction enhanced x-ray imaging. Phys Med Biol 1997;42:2015-25.

[9] Momose A, Takeda T, Itai Y, Hirano K. Phase-contrast X-Ray computed tomography for observing biological soft tissues. Nat Med 1996;2:473-5.

[10] Akisada M, Hyodo K, Ando M, Maruhashi A, Konishi K, Toyofuku F, et al. Synchrotpron Radiation at the Photon Factory for Non-Invasive Coronary Angioraphy: Experimental Studies. J Cardiography 1986;16-3:527-34.

[11] Burattini E, Gambaccini M, Marziani M, Rimondi O, Indovina PL, Pocek M, et al. X-ray mammography with synchrotron radiation. Rev Sci Instrum 1992;63: 638-40.

[12] Elleaume H, Charvet AM, Berkvens P, Berruyer G, Brochard T, Dabin Y, et al. Instrumentation of the ESRF Medical Imaging Facility. Nucl Instrum Methods Phys ResA 1999;428:513-27.

[13] Umetani K., N. Yagi, Y. Suzuki, Y. Kohmura, K. Yamasaki, X-ray refractioncontrast imaging using synchrotron radiation at SPring-8, 1999. Proceedings of SPIE, Physics of Medical Imaging 3659.

[14] Schneider P, Mohanm N, Stampanoni M, Muller R. Soft-Tissue and Phase-Contrast Imaging at the Swiss Light Source. Proc: SPIE; 2004. p. 5368.

[15] Thomlinson W, Suortti P, Chapman D. Recent advances in synchrotron radiation medical research. Nucl Instrum Methods Phys ResA 2005;543:288-96.

[16] Stevenson AW, Mayo SC, Hausermann D, Maksimenko A, Garrett RF, Hall CJ, et al. First experiments on the Australian Synchrotron Imaging and Medical beamline, including investigations of the effective source size in respect of X-ray imaging. J Synch Radiat 2010;17:75-80.

[17] Chen RC, Xie HL, Longo R, Castelli E, Xiao TQ. Opt Lett 2011;36:1719-21.

[18] Suortti P, Thomlinson W. Medical applications of synchrotron radiation. Phys Med Biol 2003;48:R1-35.

[19] Olivo A, Speller R. Modelling of a novel X-Ray phase contrast imaging technique based on coded apertures. Phys Med Biol 2007;52:6555.

[20] Ando M, Maksimenko A, Sugiyama H, Pattanasiriwisawa W, Hyodo K, Uyama C. Simple X-ray dark-and bright-field imaging using achromatic laue optics. Jpn J Appl Phys 2002;41:L1016-8.

[21] Ando M., J. Chen, K. Hyodo, K. Mori, H. Sugiyama, D. Xian and X. Zhang, Nondestructive Visual Search for Fossils in Rock Using X-Ray Interferometry Imaging, Jpn. J. Appl. Phys. 39, No.10A, L1009-L1011.

[22] Ando M, Sugiyama H, Xiaowei Z, Hyodo K, Maksimenko A, Pattanasiriwisawa W. An X-ray trichrome imaging 'trinity': absorption, phase-interference and angleresolved contrast. Jpn J Appl Phys 2002;40(3B):L298-301.

[23] Ando M, Sugiyama H, Maksimenko A, Pattanasiriwisawa W, Hyodo K, Xiaowei Z. A new optics for dark field imaging in X-ray region 'Owl'. Jpn J Appl Phys 2001; 40(8A):L844-6.

[24] Ando M., K. Hyodo, H. Sugiyama, A. Maksimenko, W. Pattanasiriwisawa, K. Mori, J. Roberson, E. Rubenstein and Y. Tanaka, 2002. X-Ray Optics 'Owl' and 'Trinity', Jpn. J. Appl. Phys. 41, No.7A, 15 July, 4742-4749.

[25] Ando M., Y. Nakao, G. Jin, H. Sugiyama, N. Sunaguchi, Y. Sung, Y. Suzuki, Y. Sun, M. Tanimoto, K. Kawashima, T. Yuasa, K. Mori, S. Ichihara, and R. Gupta, 2020 Improving contrast and spatial resolution in crystal analyzer-based X-ray darkfield imaging: Theoretical considerations and experimental demonstration, Medical Physics, doi: 10.1002/mp.14442, Aug 08. PMID: 32770681. Online ahead of print.

[26] Momose A. Phase-sensitive imaging and phase tomography using X-ray interferometers. Opt Express 2003;11:2303-14.

[27] Takeda T, Momose A, Yu Q, Wu J, Hirano K, Itai Y. Phase-contrast X-ray imaging with a large monolithic X-ray interferometer. J Synch Radiat 2000;7:280-2.

[28] Yoneyama A, Takeda T, Tsichiya Y, Wu J, Thet-Thet-Lwin A, Koizumi K, et al. A phase-contrast X-ray imaging system - with a $60 \times 30 \mathrm{~mm}$ field of view-based on a skew-symmetric two-crystal X-ray interferometer. NIM A 2004;523:217-22.

[29] Wilkins WS, Gureyev TE, Gao D, Pogany A, Stevenson AW. Phase-contrast imaging using polychromatic hard X-rays. Nature 1996;384:335-8.

[30] Gureyev TE, Mayo SC, Myers DE, Nesterets Y, Paganin DM, Pogany A, et al. Refracting Röntgen's rays: Propagation-based x-ray phase contrast for biomedical imaging. J Appl Phys 2009;105:102005.

[31] Momose A, Kawamoto S, Koyama I, Hamaishi Y, Takai K, Suzuki Y. Demonstration of X-ray talbot interferometry. Jpn J Appl Phys 2003;42:L866-8.

[32] Pfeiffer F, Bech M, Bunk O, Kraft P, Eikenberry EF, Brönnimann C, et al. Hard-Xray dark-field imaging using a grating interferometer. Nat Mater 2008;7:134-7. 
[33] Sunaguchi N, Yuasa T, Huo Q, Ichihara S, Ando M. X-ray refraction-contrast computed tomography images using dark-field imaging optics. Appl Phys Lett 2010;97:153701.

[34] Ando M, Sunaguchi N, Shimao D, Pan A, Yuasa T, Mori K, et al. Dark-field imaging: recent developments and potential clinical applications. Phys Med 2016 32:1801-12.

[35] Pacile S, Baran P, Dullin C, Dimmock M, Locked D, Missback-Guntner J, et al. Advantages of breast cancer visualization and characterization using synchrotron radiation phase-contrast tomography. J Synch Radiat 2018;25:1460-6.

[36] Arboleda C, Wang Z, Jefimovs K, Koehler T, Van Stevendaal U, Kuhn N, et al Towards clinical grating-interferometry mammography. Eur Radiol 2020;30: $1419-25$.

[37] Authier A. Dynamical theory of X-Ray diffraction. Oxford: Oxford Science Publications; 2001.

[38] Kikuta S. X-Ray Scattering and Synchrotron Radiation Science Basic (in Japanese)". University of Tokyo Press; 2011.

[39] Takagi S. Dynamical theory of diffraction applicable to crystals with any kind of small distortion. Acta Crystallogr A 1962;15(12):1311-2.

[40] Taupin D., 1964. Dynamic theory of x-ray diffraction in crystals. Bull. Soc. Fr. Mineral. Crystallogr. 87.

[41] Sanchez del Rio M. and R. J. Dejus, 2011. Status of XOP: v2.4: recent developments of the x-ray optics software toolkit, SPIE Proceedings 8141, pp.814115 doi:10.1117/12.893911.

[42] Ando M, Sugiyama H, Ichihara S, Endo T, Bando H, Yamasaki K, et al. Sharper image of breast cancer cells and stroma in the dense breast by thinner angular filter under X-Ray dark-field imaging. Jpn J Appl Phys 2006;45(28):L740-3.

[43] Suzuki Y., Y. Chikaura and M. Ando, 2011. Computer Simulation on Spatial Resolution of X-ray Bright-Field Imaging by Dynamical Diffraction Theory for a Laue-case Crystal Analyzer, J. Appl. Phys. 110, 084902-1 084902-4.

[44] Hirano K., A. Maksimenko, H. Sugiyama and M. Ando, 2002. X-Ray Optics for Observing Dark-Field and Bright-Field Refraction-Contrast Images, Jpn. J. Appl. Phys. 41, Part 2, No. 5B, L595-L598.

[45] Maksimenko A, Ando M, Sugiyama H, Yuasa T. Computed tomographic reconstruction based on refraction contrast. Appl Phys Lett 2005;86:124105.

[46] Yuasa T, Maksimenko A, Hashimoto E, Sugiyama H, Hyodo K, Akatsuka T, et al. Hard-x-ray region tomographic reconstruction of the refractive-index gradient vector field: imaging principles and comparisons with diffraction-enhancedimaging-based computed tomography. Opt Lett 2006;31:1818-20.

[47] Sunaguchi N, Yuasa T, Huo Q, Ando M. Convolution reconstruction algorithm for refraction-contrast computed tomography using a Laue-case analyzer for dark field imaging. Opt Lett 2011;36:391-3.

[48] Sunaguchi N, Yuasa T, Ando M. Iterative reconstruction algorithm for analyzerbased phase-contrast computed tomography of hard and soft tissue. Appl Phys Lett 2013;103:143702.

[49] Sunaguchi N, Yuasa T, Sun F, Gupta R, Ando M. Limited view reconstruction for differential phase-contrast computed tomography. Opt Express 2015;23:9717-29.

[50] Sunaguchi N, Yuasa T, Hirano S, Gupta R, Ando M. In vitro validation of an artefact suppression algorithm in X-ray phase-contrast computed tomography PLOS ONE 2015;10:e0122347.

[51] Sunaguchi N, Yuasa T, Gupta R, Ando M. An efficient reconstruction algorithm for differential phase-contrast tomographic images from a limited number of views Appl Phys Lett 2015;107:253701.

[52] Sunaguchi N, Yuasa T, Huo Q, Ichihara S, Ando M. X-ray refraction-contrast computed tomography images using dark field imaging optics. Appl Phys Lett 2010;97(15):153701.

[53] Nakane PK, Pierce Jr GB. Enzyme-labeled antibodies for the light and electron microscopic localization of tissue antigens. J Cell Biol 1967;33(2):307-18.

[54] Coons AH, Creech HJ, Jones RN. Immunological Properties of an Antibody Containing a Fluorescent Group. Exp Biol Med 1941;47(2):200-2.

[55] Marcum RG, Wellings SR. Subgross pathology of the human breast: method and initial observations. J Natl Cancer Inst 1969;42(1):115-21.

[56] Takahashi T. Lobular Structure of the Human Liver from the Viewpoint of Hepatic Vascular Architecture. J Exp Med 1970;101(2):119-40.

[57] Wellings SR, Jensen HM. On the origin and progression of ductal carcinoma in the human breast. J Natl Cancer Inst 1973;50(5):1111-8.

[58] Wellings SR, Jensen HM, Marcum RG. An atlas of subgross pathology of the human breast with special reference to possible precancerous lesions. J Natl Cancer Inst 1975;55(2):231-73.

[59] Norton KA, Namazi S, Barnard N, Fujibayashi M, Bhanot G, Ganesan S, et al. Automated reconstruction algorithm for identification of 3D architectures of cribriform ductal carcinoma in situ. PLoS ONE 2012;7(9):1-11.

[60] Magee D, Song Y, Gilbert S, Roberts N, Wijayathunga N, Wilcox R, et al. Histopathology in 3D: from three-dimensional reconstruction to multi-stain and multi-modal analysis. J Pathol Inf 2015.

[61] Roberts N, Magee D, Song Y, Brabazon K, Shires M, Crellin D, et al. Toward routine Use of 3D histopathology as a research tool. Am J Pathol 2012;180(5): $1835-42$.

[62] Booth ME, Treanor D, Roberts N, Magee DR, Speirs V, Hanby AM. Threedimensional reconstruction of ductal carcinoma in situ with virtual slides. Histopathology 2015;66(7):966-73.

[63] Song Y, Treanor D, Bulpitt AJ, Magee DR. 3D reconstruction of multiple stained histology images. J Pathol Inf 2013.

[64] Marchiò C, Sapino A, Arisio R, Bussolati G. A new vision of tubular and tubulolobular carcinomas of the breast, as revealed by 3-D modelling. Histopathology 2006;48(5):556-62.
[65] Onozato ML, Klepeis VE, Yagi Y, Mino-Kenudson M. A role of three-dimensional (3D)-reconstruction in the classification of lung adenocarcinoma. Anal Cell Pathol 2012;35(2):79-84.

[66] Webster JD, Dunstan RW. Whole-slide imaging and automated image analysis: considerations and opportunities in the practice of pathology. Vet Pathol 2014;51 (1):211-23.

[67] Komura D, Ishikawa S. Machine learning methods for histopathological image analysis. Comput Struct Biotechnol J 2018;16:34-42.

[68] Jansen I, Lucas M, Savci-Heijink CD, Meijer SL, Liem EIML, de Boer OJ, et al, Three-dimensional histopathological reconstruction of bladder tumours. Diagn Pathol 2019;14(1):25.

[69] Melo RCN, Raas MWD, Palazzi C, Neves VH, Malta KK, Silva TP. Whole slide imaging and its applications to histopathological studies of liver disorders. Front Med (Lausanne) 2020;6:310.

[70] Kimura J, Tsukise A, Yokota H, Nambo Y, Higuchi T. The application of threedimensional internal structure microscopy in the observation of mare ovary. Anat Histol Embryol 2001;30(5):309-12.

[71] Fujisaki K, Yokota H, Furushiro N, Komatani S, Ohzawa S, Sato Y. Threedimensional microscopic elemental analysis using an automated high-precision serial sectioning system. Microsc Microanal 2011;17(2):246-51.

[72] Ono M, Akuzawa H, Nambo Y, Hirano Y, Kimura J, Takemoto S, et al. Analysis of the equine ovarian structure during the first twelve months of life by threedimensional internal structure microscopy. J Vet Med Sci J 2016;77(12): 1599-603.

[73] Hashimoto H, Kusakabe M, Ishikawa H. A novel method for three-dimensional observation of the vascular networks in the whole mouse brain. Microsc Res Tech 2008;71(1):51-9.

[74] Tuchin VV. Tissue optics and photonics: light-tissue interaction. J Biomed Photon Eng 2015;1:98-134.

[75] Spalteholz W. Über das durchsichtigmachen von menschlichen und tierischen präparaten. Leipzig: S. Hirzel; 1914.

[76] Susaki EA, Ueda HR. Whole-body and whole-organ clearing and imaging techniques with single-cell resolution: toward organism-level systems biology in mammals. Cell Chem Biol 2016;23:137-57.

[77] Tainaka K, Kuno A, Kubota SI, Murakami T, Ueda HR. Chemical Principles in Tissue Clearing and Staining Protocols for Whole-Body Cell Profiling. Annu Rev Cell Dev Biol 2016;32:713-41.

[78] Tainaka K, Murakami TC, Susaki EA, Shimizu C, Saito R, Takahashi K, et al. Chemical landscape for tissue clearing based on hydrophilic reagents. Cell Rep 2018;24:2196-210.

[79] Nojima S., E.A. Susaki, K. Yoshida, H. Takemoto, N. Tsujimura, S. Iijima, K. Takachi, Y. Nakahara, A. Tahara, K. Ohshima, M. Kurashige, Y. Hori, N. Wada, J. I. Ikeda, A. Kumanogoh, E. Morii, H.R. Ueda, 2017. CUBIC pathology: threedimensional imaging for pathological diagnosis, Sci. Rep., 24, 7(1), 9269.

[80] Huisken J, Swoger J, Del Bene F, Wittbrodt J, Stelzer EH. Optical sectioning deep inside live embryos by selective plane illumination microscopy. Science 2014;305 (5686):1007-9.

[81] Tainaka K, Kubota SI, Suyama TQ, Susaki EA, Perrin D, Ukai-Tadenuma M, et al. Whole-body imaging with single-cell resolution by tissue decolorization. Cell 2014;159(4):911-24.

[82] Susaki EA, Shimizu C, Kuno A, Tainaka K, Li X, Nishi K, et al. Versatile wholeorgan/body staining and imaging based on electrolyte-gel properties of biological tissues. Nat Commun 2020;11:1982.

[83] Webb S, Flower MA. Webb's physics of medical imaging. London: CRC Press; 2012.

[84] Intl Commission on Radiation, 1992. Photon, electron, proton and neutron interaction data for body tissues., ICRU Report, 46.

[85] Ando M, Sunaguchi N, Wu Y, Do S, Sung Y, Louissaint A, et al. Crystal analyserbased X-ray phase contrast imaging in the dark field: implementation and evaluation using excised tissue specimens. Eur Radiol 2014;24:423-33. https:// doi.org/10.1007/s00330-013-3021-9.

[86] Schroeder S, Kopp AF, Baumbach A, Meisner C, Kuettner A, Georg C, et al. Noninvasive detection and evaluation of atherosclerotic coronary plaques with multislice computed tomography. J Am Coll Cardiol 2001;37(5):1430-5.

[87] Chu B, Kampschulte A, Ferguson MS, Kerwin WS, Yarnykh VL, O’Brien KD, et al. Hemorrhage in the atherosclerotic carotid plaque: a high-resolution MRI study. Stroke 2004;35(5):1079-84.

[88] Goehde SC, Hunold P, Vogt FM, Ajaj W, Goyen M, Herborn CU, et al. Full-body cardiovascular and tumor MRI for early detection of disease: feasibility and initial experience in 298 subjects. AJR Am J Roentgenol 2005;184(2):598-611.

[89] Rawson SD, Maksimcuka J, Withers PJ, Cartmell SH. X-ray computed tomography in life sciences. BMC Biol 2020;18(1):21.

[90] Maire E, Withers PJ. Quantitative X-ray tomography. Int Mater Rev 2014;59(1): $1-43$.

[91] Ichihara S, Ando M, Maksimenko A, Yuasa T, Sugiyama H, Hashimoto E, et al. 3-D reconstruction and virtual ductoscopy of high-grade ductal carcinoma in situ of the breast with casting type calcifications using refraction-based X-ray CT. Virchows Arch 2008;452:41-7. https://doi.org/10.1007/s00428-007-0528-y.

[92] Kao T, Connor D, Dilmanian FA, Faulconer L, Liu T, Parham C, et al. Characterization of diffraction-enhanced imaging contrast in breast cancer. Phys Med Biol 2009;54(10):3247-56.

[93] Sunaguchi N, Shimao D, Yuasa T, Ichihara S, Nishimura R, Oshima R, et al. Threedimensional microanatomy of human nipple visualized by X-ray dark-field computed tomography. Breast Cancer Res Treat 2020;180(2):397-405. 
[94] Sunaguchi N, Shimao D, Ichihara S, Mori K, Yuasa T, Ando M. Three-dimensional reconstruction of human nipple using refraction-contrast X-ray computed Tomography. AIP Conf Proc 2019;2054:050010.

[95] Pisano ED, Johnston RE, Chapman D, Geradts J, Iacocca MV, Livasy CA, et al. Human breast cancer specimens: diffraction-enhanced imaging with histologic correlation-improved conspicuity of lesion detail compared with digital radiography. Radiology 2000;214:895-901. https://doi.org/10.1148/ radiology.214.3.r00mr26895.

[96] Fiedler S, Bravin A, Keyriläinen J, Fernández M, Suortti P, Thomlinson W, et al. Imaging lobular breast carcinoma: comparison of synchrotron radiation DEI-CT technique with clinical CT, mammography and histology. Phys Med Biol 2004;49: 175-88. https://doi.org/10.1088/0031-9155/49/2/001.

[97] Keyrilainen J, Fernandez M, Fiedler S, Bravin A, Karjalainen-Lindsberg ML, Virkkunen $\mathrm{P}$, et al. Visualisation of calcifications and thin collagen strands in human breast tumour specimens by the diffraction-enhanced imaging technique: a comparison with conventional mammography and histology. Eur J Radiol 2005; 53:226-37. https://doi.org/10.1016/j.ejrad.2004.03.015.

[98] Bravin A, Keyrilainen J, Fernandez M, Fiedler S, Nemoz C, KarjalainenLindsberg ML, et al. High-resolution CT by diffraction-enhanced $x$-ray imaging: mapping of breast tissue samples and comparison with their histo-pathology. Phys Med Biol 2007;52:2197-211. https://doi.org/10.1088/0031-9155/52/8/ 011.

[99] Keyrilainen J, Fernandez M, Karjalainen-Lindsberg ML, Virkkunen P, Leidenius M, von Smitten K, et al. Toward high-contrast breast CT at low radiation dose. Radiology 2008;249:321-7. https://doi.org/10.1148/ radiol.2491072129.

[100] Ando M., H. Bando, T. Endo, S. Ichihara, E. Hashimoto, K. Hyodo, T. Kunisada, G. Li, A. Maksimenko, K. Mori, D. Shimao, H. Sugiyama, T. Yuasa, E. Ueno, 2008. Refraction-based 2D, 2.5D and 3D medical imaging: stepping forward to a clinical trial. Eur. J. Radiol 68, S32-S36. https : //doi.org/10.1016/j.ejrad.2008.04.033.

[101] Sunaguchi N, Shimao D, Yuasa T, Ichihara S, Nishimura R, Oshima R, et al. Threedimensional microanatomy of human nipple visualized by X-ray dark- field computed tomography. Breast Cancer Res Treat 2020;180:397-405.

[102] Going JJ, Moffat DF. Escaping from Flatland: clinical and biological aspects of human mammary duct anatomy in three dimensions. J Pathol 2004;203:538-44.

[103] Rusby JE, Brachtel EF, Michaelson JS, et al. Breast duct anatomy in the human nipple: three-dimensional patterns and clinical implications. Breast Cancer Res Treat 2007;106(2):171-9.

[104] Tan MP, Tot T. The sick lobe hypothesis, field cancerisation and the new era of precision breast surgery. Gland Surg 2018;27:611-8.

[105] Koerner F, Maluf H. Uncommon morphologic patterns of lobular neoplasia. Ann Diagn Pathol 1999;3:249-59.
[106] Albers J, Pacile S, Markus MA, et al. X-ray-Based 3D Virtual Histology-Adding the Next Dimension to Histological Analysis. Mol Imaging Biol 2018;20:732-41.

[107] Dobbins III JT, Godfrey DJ. Digital x-ray tomosynthesis: current state of the art and clinical potential. Phys Med Biol 2003;48:R65-106.

[108] Shimao D, Kunisada T, Sugiyama H, Ando M. Refraction Enhanced Tomosynthesis of Finger Joint by X-Ray Dark-Field Imaging. Jpn J Appl Phys 2007;46:L608-10.

[109] Shimao D, Kunisada T, Sugiyama H, Ando M. Shift-and-add tomosynthesis of a finger joint by X-ray dark-field imaging: difference due to tomographic angle. Eur J Radiol 2008;68S:S27-31.

[110] Shimao D, Mori K, Sugiyama H, Hyodo K. Imaging of ligament and articular cartilage due to refraction-contrast using a Laue geometry analyzer crystal. Jpn J Appl Phys 2003;42:5874-5.

[111] Shimao D, Sugiyama H, Hyodo K, Kunisada T, Ando M. Evaluation of X-ray darkfield imaging in visualization of nearly clinical articular cartilage. Nucl Instrum Meth A 2005;548:129-34.

[112] Shimao D, Sugiyama H, Hyodo K, Kunisada T, Ando M. Articular cartilage depicted at optimized angular position of Laue angular analyzer by X-ray darkfield. Appl Radiat Isot 2006;64:868-74.

[113] Ando M, N. Sunaguchi, S. Yongjin, J.-K. Kim, G. Li, Y. Suzuki, T. Yuasa, K. Mori, S. Ichihara, and R. Gupta 2018. Crystal-based X-ray Medical Imaging Using Synchrotron Radiation and Its Future Prospect, Synchrotron Radiation Applications 287-341, ed. XinYi Zhang, World Scientific Publisher.

[114] Seo S-J, Sunaguchi N, Ando M, Choi K-H, Kim H, Chang W-S, et al. Visualization of microvessel proliferation as a tumor infiltration structure in rat glioma specimens using the diffraction-enhanced imaging in-plane CT technique. PMB 2012;57:1251-62.

[115] Feser M., R. Ruth, R. Lowen and J. Kasahara, 2018, Bridging the Synchrotron @Lab Source Gap for Microscopy : The Inverse Compton Scattering X-ray Source, Proceedings of the 14th International Conference on X-ray Microscopy (XRM2018), 24, 306-309.

[116] Sato H, Shimao D, Ando M. Investigation of absorbed radiation dose in refractionenhanced breast tomosynthesis by a laue case analyzer. Radiat Prot Dosim 2011; $145: 1-3$.

[117] Brombal L, Arfelli F, Delogu P, Donato S, Mettivier G, Michieldsen K, et al. Image quality comparison between a phase-constrast synchrotron radiation breast CT and a clinical breast CT: a phantom based study. Sci Rep Nat Res 2019;9:17778. https://doi.org/10.1038/s41598-019-54131-z.

[118] Saccomano M, Albers J, Tromba G, Dobrivojevic Radmilovic M, Gajovic S, Alves F, et al. Synchrotron inline phase contrast $\mu \mathrm{CT}$ enables detailed virtual histology of embedded soft-tissue samples with and without staining. J Synchrotron Radiat 2018;25:1153-61. https://doi.org/10.1107/ S1600577518005489. 